\title{
Non-Destructive Evaluation of Materials Using Pulsed Microwave Interrogating Signals and Acoustic Wave Induced Reflections
}

\author{
R. A. Albanese \\ Human Effectiveness Directorate \\ Biomechanisms and Modeling Branch \\ AFRL \\ Brooks AFB, TX 78235 \\ H. T. Banks and J. K. Raye \\ Center for Research in Scientific Computation \\ North Carolina State University \\ Raleigh, NC 27695-8205 \\ email: htbanks@eos.ncsu.edu
}

April 28, 2002

\begin{abstract}
A class of inverse problems is formulated for the estimation of material dielectric parameters using reflections of pulsed microwave interrogating signals from moving acoustic interfaces in the dielectric. A model for acoustic pressure-dependence of material constitutive parameters in Maxwell's equations is presented and analyzed. Theoretical and computational findings for approximation and estimation are given.
\end{abstract}




\section{Introduction and problem formulation}

An increasing number of papers in electromagnetic material interrogation are oriented toward nondestructive or even noninvasive detection of material properties. Among these are dielectric properties, such as permittivity and conductivity, and/or geometry, including size, shape, and location of occlusions. For example, in subsurface damage detection via eddy current techniques (see [7], [5], and [6]) the goal is to identify regions of low or zero conductivity which represent voids or barriers to electrical conductivity as appear in delaminations, corrosions, and noncontacting cracks. The subsurface or internal structural interrogation we focus on here depends less on the conductivity of the material than on the material electric polarization. Such techniques are therefore applicable in non-invasive examination of tissue, which is a low conductive dielectric, to detect such anomalies as abnormalities in cell structure and chemical composition as well as in underground detection of mines and bunkers containing rebar concrete with highly conductive components.

There are several ways in which these polarization based interrogation techniques may be attempted. One may discern material properties by observing electromagnetic microwave impulses after they have been transmitted through the target (see the discussions and references in Chapter 1 of [4] and [1]). Difficulties for this approach include large and/or highly dissipative and dispersive material targets as well as the infeasibility of placing sensors on the back side of the target.

A second approach entails the interrogation via reflections from a target with a highly conductive back boundary. Examples include use of a metal tipped catheter (this, however, scarcely qualifies as noninvasive!) in tissue regions that are accessible, an artery for instance, or targets with metal backed surfaces, such as aircraft, or metal containing structures including rebar concrete and communication equipment. However widespread use of such techniques is not feasible in many targets, especially in biomedical diagnostics involving in vivo interrogation.

A third possibility, treated in this paper, entails use of a traveling acoustic wave as a reflecting virtual interface for propagating microwave impulses. It is rather well-accepted (e.g., see [22]) that acoustic pressure waves will interact with electromagnetic signals in ways that often mimic interfacial partial reflection/partial transmission for the electromagnetic waves. In [4] the authors investigated aspects of elementary electromagnetic/acoustic wave interaction. The modeling presented in [4] was rather naive and not based on any specific physical mechanisms. Moreover, a fixed (standing) acoustic wave was employed as the reflecting interface. In this paper, we continue the investigations begun in [4] where a remote antenna generates a pulsed microwave interrogating signal that reflects from natural material interfaces. Here we attempt to develop both theory and computation to demonstrate that one can use reflections from both boundary interfaces and traveling acoustic-generated interfaces to estimate polarization properties in dielectric material targets. In order to accomplish this, 
one must have a viable model for acoustic pressure-dependent material polarization. In Section 2, we present such a polarization model founded on accepted mechanisms. Our model is based on the Debye model for orientational polarization. We modify this model to incorporate pressure-dependence and allow for the electromagnetic/acoustic interaction that results in the reflections. Other approaches to modeling electromagnetic/acoustic interaction are outlined in [10]. In Section 3, we develop a theoretical framework for the inverse problem which underlies the interrogation technique. Finally in Section 4, we provide sample inverse problem calculations to support the feasibilty of the technique.

Under certain assumptions (see also [4]), including material homogeneity in directions perpendicular to the direction of electromagnetic wave propagation and the use of a polarized planar electromagnetic wave, we may model the interrogation technique using a one dimensional form of Maxwell's equations with temporally and spatially varying coefficients. Provided that these assumptions hold, Maxwell's equations can be written

$$
\begin{aligned}
\frac{\partial}{\partial z} E & =-\mu_{0} \frac{\partial}{\partial t} H \\
\frac{\partial}{\partial z} H & =\frac{\partial}{\partial t} D+\sigma E+J_{s}
\end{aligned}
$$

where $E$ and $H$ are the electric and magnetic fields, $D$ is the electric flux density, and $J_{s}$ is the source current density. The macroscopic polarization $P$ is introduced via the electric flux density through the relation

$$
D=\epsilon_{0} \epsilon_{r} E+P
$$

where $\epsilon_{r}$ represents the effects of instantaneous polarization in the dielectric. We discuss the form of $P$ in great detail in Section 2. By taking the appropriate derivatives of (1) and (2) and employing equation (3), we obtain

$$
\mu_{0} \epsilon_{r} \ddot{E}+\mu_{0} \ddot{P}+\mu_{0} \sigma \dot{E}-E^{\prime \prime}=-\mu_{0} \dot{J}_{s} .
$$

Here and throughout we use $\dot{E}$ to denote $\frac{\partial}{\partial t} E$ and $E^{\prime}$ to denote $\frac{\partial}{\partial z} E$.

We consider (4) in the domain $0 \leq z \leq 1$ with air in the interval $\left[0, z_{1}\right]$ and a dielectric material in the region $\left(z_{1}, 1\right]\left(0<z_{1}<1\right)$. We enforce an absorbing boundary condition on the left $(z=0)$ and a perfectly conductive boundary condition on the right $(z=1)$. We leave the initial conditions for the electric field in general form, but we assume that the polarization and its first time derivative are initially zero (this may be done without loss of generality). The geometry of the problem is shown in Figure 1. 


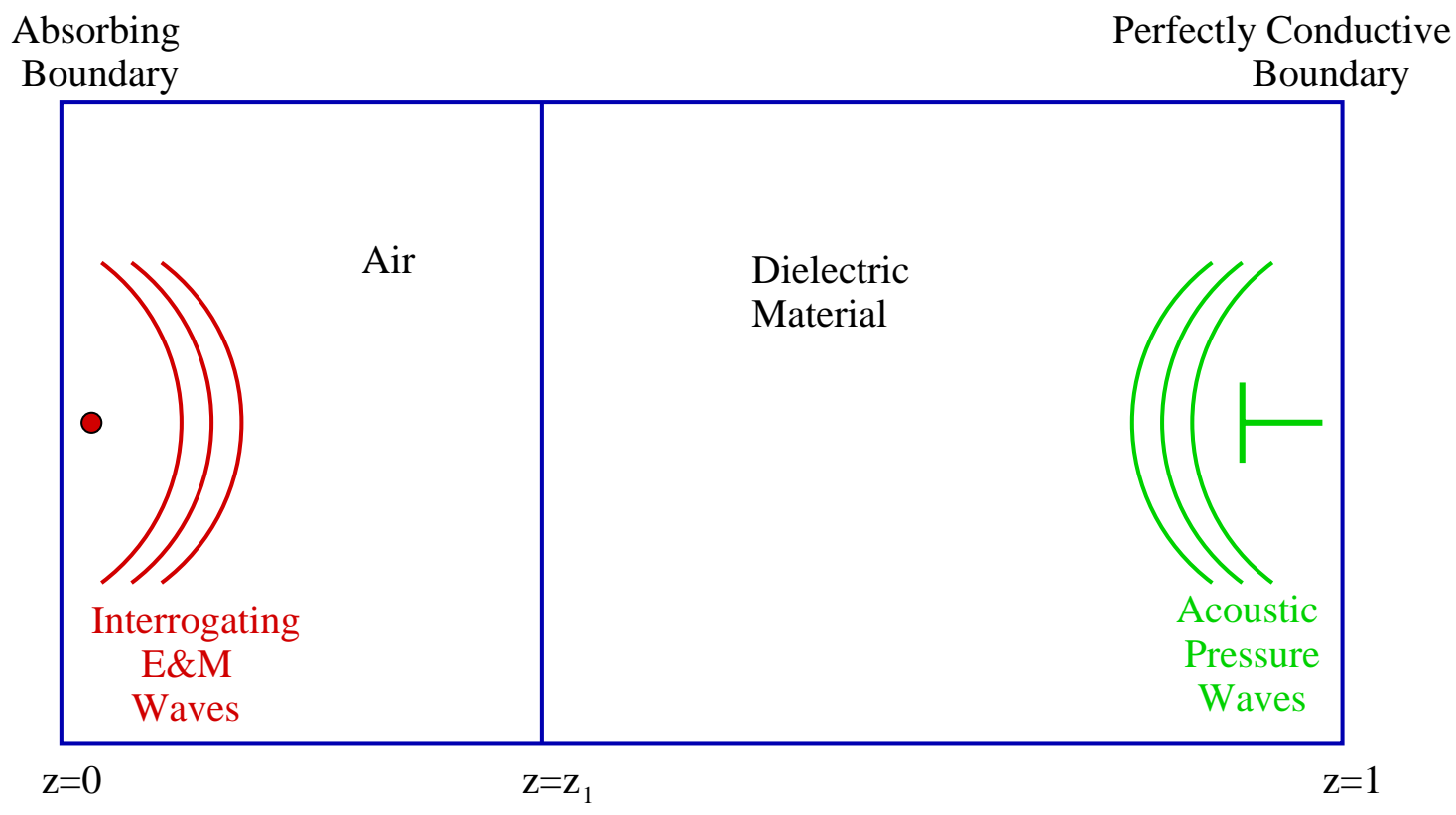

Figure 1: Schematic of geometry

\section{Polarization model}

In this section, we present and motivate our model for pressure-dependent polarization. We begin by discussing polarization in general and then explain how it pertains to our problem.

\subsection{Mechanisms of polarization}

Electric polarization is by definition the electric dipole moment per unit volume. The formation of these electric dipoles can be caused by several mechanisms [2], [3] which we briefly summarize here.

Electronic polarization/ Optical polarization/ Induced polarization An applied field displaces the electron cloud center of an atom with respect to its nucleus. This induces a dipole moment. Electronic polarization is found in both materials that possess molecules with large dipole moments (polar materials) and those that do not (nonpolar materials).

Atomic polarization/ Ionic polarization/ Molecular polarization An applied electric field may displace the atoms in the molecules, changing the distance between the atoms, and thus changing the dipole moment. Atomic polarization only occurs in polar materials. 
Orientational polarization/ Dipole polarization Without an applied field, a polar material possesses permanent dipole moments that are randomly oriented. When a field is applied, these dipoles align themselves with the field. Since orientational polarization is reliant upon the existence of permanent dipole moments, it only is found in polar materials.

Interfacial polarization The impurities and defects in crystal can impede the flow of charge created by an applied field. The resulting charge accumulation can result in a dipole moment. This type of polarization is found only in crystals.

The multiple names for each type of polarization can be confusing, especially when comparing the research of different contributors. We attempt to refer to each mechanism by the first name given above. We point out that in addition to this terminology both atomic and electronic polarization are sometimes referred to as distortional polarization [2].

In a given material, polarization can be the result of one or more of these four mechanisms. We are primarily interested in materials that contain a high water-content, such as living tissue or mud, so here we focus on polar liquids. The polarization in this class of liquids tends to depend mostly on the orientation of permanent electric dipoles in the molecules (orientational polarization) and the distortion of the molecules by an applied electric field (electronic and atomic polarization) [16]. With this in mind, we focus on these polarization mechanisms in the remainder of our discussions.

In the presence of most applied electric fields, the polarization of a high water-content liquid is both distortional and orientational. At high (optical) frequencies however, the electric field oscillates so rapidly that it does not hold any orientation long enough for the dipoles to align with it. Thus, the orientational polarization is virtually insignificant [26]. This implies that at sufficiently high frequencies, the only contribution to the dielectric constant or optical index of refraction is from electrical distortion [16].

Since the polarization of a polar liquid has multiple mechanisms, we expect that a complete model must incorporate them all. Orientational polarization is suggestive of a mechanism with an exponential decay factor, such as the one in the model proposed by Debye [2]. However, a system rarely conforms exactly to the model described by the Debye dispersion equations due to the fact that the polarizational decay may not be represented accurately by a mechanism with one relaxation time [17]. On the other hand, distortional polarization causes charges to behave somewhat like linear harmonic oscillators; thus it is reasonable to model them as such (the Lorentz model is an example). Neither of these types of models alone will be sufficient to completely describe the polarization of a polar liquid. Nonetheless as a first step and to illustrate our ideas, we base our model on the Debye model for orientational polarization. Future modeling attempts will require systems of more complexity. 


\subsection{The Debye model}

The Debye model [4] can be represented by the first order ordinary differential equation

$$
\tau \dot{P}+P=\epsilon_{0}\left(\epsilon_{s}-\epsilon_{\infty}\right) E
$$

or by

$$
P(t, z)=\int_{0}^{t} g(t-s, z) E(s, z) d s
$$

with kernel

$$
g(t)=\exp \left(\frac{-t}{\tau}\right) \frac{\epsilon_{0}\left(\epsilon_{s}-\epsilon_{\infty}\right)}{\tau}
$$

In these equations, $\epsilon_{s}$ is the static relative permitivitty and $\epsilon_{\infty}$ is the value of permittivity for an extremely high $(\approx$ infinite) frequency field. In this model, the value of the relative permittivity $\epsilon_{r}$ of (3) in the dielectric is given by $\epsilon_{\infty}$; that is, $\epsilon_{r}=1$ in $\left[0, z_{1}\right]$ and $\epsilon_{r}=\epsilon_{\infty}$ in $\left(z_{1}, 1\right]$. The variable $\tau$ is the relaxation time of the dielectric.

In [2], Anderson describes a potential double well formulation for an atomic model that leads to the Debye polarization model. In this model, the dielectric is made up of independent noninteracting particles; each particle has two equilibrium positions separated by a barrier of high potential. One considers a charged particle with two equilibrium positions $A$ and $B$ located a distance $d$ from each other. Between them is a potential barrier $W$ such that $W \gg k_{B} T$ where $k_{B}$ is the Boltzmann constant and $T$ is the temperature. (See Figure 2.) If there is no electromagnetic field present, one assumes that the particle oscillates about either equilibrium, and on occassion, obtains enough energy to cross the potential barrier and jump into the other well. Over time, for constant temperature, the particle is near $A$ as often as near $B$ and the probability of finding the particle near a given well is $\frac{1}{2}$. 

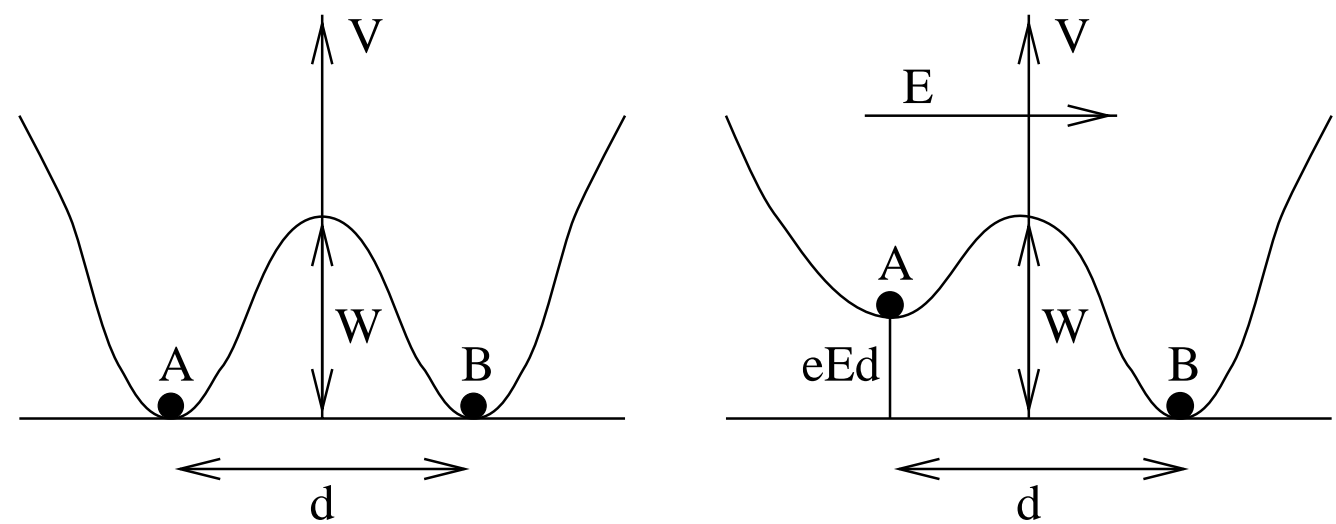

Figure 2: Potential double well model with and without an applied field

When an electric field $E$ is applied in the direction from $A$ to $B$, the potentials at each equilibrium are no longer equal, for instance $V_{A}>V_{B}$, and

$$
V_{A}-V_{B}=e d E
$$

where $e$ is the charge of the particle. (See Figure 2.) A result from Boltzmann statistics implies that the probability that a particle has potential $V$ is proportional to $\exp \left(-\frac{V}{k_{B} T}\right)$, so that now it is more likely to find the particle near equilibrium $B$. As before, a particle can jump from one equilibrium to the other if it acquires enough energy. For a potential barrier $W$, the probability that a particle can cross this barrier in the direction from $B$ to $A$ is proportional to $\exp \left(-\frac{W}{k_{B} T}\right)$, with proportionality constant $\frac{w_{0}}{2 \pi}$, the assumed frequency of oscillation due to thermal agitation of the particle about the equilibrium. Likewise, the probability that the particle can cross the barrier in the direction from $A$ to $B$ is given by $\frac{w_{0}}{2 \pi} \exp \left(-\frac{W-e d E}{k_{B} T}\right)$. Using these probabilities and the fact that the total number $N=N_{A}+N_{B}$ of particles is constant, one can derive [2] (see also page 387 of [15]) a linear first order differential equation to describe the difference $N_{B}(t)-N_{A}(t)$ in number of particles in wells $B$ and $A$ at any time $t$

$$
\begin{gathered}
\frac{d}{d t}\left(N_{B}(t)-N_{A}(t)\right)= \\
\frac{w_{0}}{\pi} \exp \left(-\frac{W}{k_{B} T}\right)\left(-\left(N_{B}(t)-N_{A}(t)\right)+\frac{e d}{2 k_{B} T} N E\right) .
\end{gathered}
$$

The polarization $P(t)$ due to the applied electromagnetic field is proportional to $N_{B}(t)-$ $N_{A}(t)$. By relating $\tau$ with $\frac{w_{0}}{\pi} \exp \left(-\frac{W}{k_{B} T}\right)$ and $\epsilon_{s}-\epsilon_{\infty}$ with $\frac{w_{0}}{\pi} \exp \left(-\frac{W}{k_{B} T}\right) \frac{e d}{2 k_{B} T} N$, one thus arrives at the Debye model (5) from atomic considerations. 
There is substantial reason to believe that the behavior described by the Debye model is pressure-dependent. One approach to understanding this pressure-dependence is to extend the above arguments and consider the polarization from a non-equilibrium thermodynamics perspective. A discussion of this nature is given in [20].

We however take a different approach to incorporating pressure-dependence into the Debye model. We present the model here and provide motivation in Section 2.3. We begin by assuming that the material-dependent parameters in the differential equation (5) depend on pressure, i.e.,

$$
\tau(p) \dot{P}+P=\epsilon_{0}\left(\epsilon_{s}(p)-\epsilon_{\infty}(p)\right) E=\epsilon_{0}(\gamma(p)-\zeta(p)) E .
$$

We suppose as a first approximation that each of the pressure-dependent parameters can be represented as a mean value plus a perturbation that is proportional to the pressure

$$
\begin{aligned}
\tau(p) & =\tau_{0}+\tilde{\tau}=\tau_{0}+\kappa_{\tau} p \\
\gamma(p) & =\gamma_{0}+\tilde{\gamma}=\gamma_{0}+\kappa_{\gamma} p \\
\zeta(p) & =\zeta_{0}+\tilde{\zeta}=\zeta_{0}+\kappa_{\zeta} p .
\end{aligned}
$$

Then the equation

$$
\tau(p) \dot{P}+P=\epsilon_{0}(\gamma(p)-\zeta(p)) E
$$

can be written

$$
\left(\tau_{0}+\kappa_{\tau} p\right) \dot{P}+P=\epsilon_{0}\left(\gamma_{0}-\zeta_{0}+\left(\kappa_{\gamma}-\kappa_{\zeta}\right) p\right) E .
$$

We recall that the polarization term in (4) involves second-order time derivatives. To express (6) in compatible form, we take the time derivative of both sides to obtain

$$
\ddot{P}=-\frac{\left(1+\kappa_{\tau} \dot{p}\right)}{\left(\tau_{0}+\kappa_{\tau} p\right)} \dot{P}+\frac{\epsilon_{0}\left(\gamma_{0}-\zeta_{0}+\left(\kappa_{\gamma}-\kappa_{\zeta}\right) p\right)}{\left(\tau_{0}+\kappa_{\tau} p\right)} \dot{E}+\frac{\epsilon_{0}\left(\kappa_{\gamma}-\kappa_{\zeta}\right) \dot{p}}{\left(\tau_{0}+\kappa_{\tau} p\right)} E
$$

with

$$
\dot{P}=-\frac{1}{\left(\tau_{0}+\kappa_{\tau} p\right)} P+\frac{\epsilon_{0}\left(\gamma_{0}-\zeta_{0}+\left(\kappa_{\gamma}-\kappa_{\zeta}\right) p\right)}{\left(\tau_{0}+\kappa_{\tau} p\right)} E .
$$


From here, we can use (8) in (7) and then replace $\ddot{P}$ in (4) with the expression given by (7).

Additionally, we should note that the relation $\epsilon_{r}=\epsilon_{\infty}$ in $\left(z_{1}, 1\right]$ becomes $\epsilon_{r}=\epsilon_{\infty}(p)=\zeta_{0}+\kappa_{\zeta} p$ in $\left(z_{1}, 1\right]$.

\subsection{Motivation for pressure-dependence of polarization}

The polarization described by both the original and pressure-dependent Debye models is due to the alignment of permanent dipole moments with the applied field. This tendency to align is inhibited by the presence of centrifugal or gyroscopic forces. These forces are caused by molecular rotations and collisions [26]. The pressure in a polarizable medium influences and is influenced by the short range particle interaction in the medium, including these molecular rotations and collisions. This interaction between particles may serve to inhibit or facilitate the alignment of dipole moments with the applied field, resulting in the modification of the orientational polarization [14]. Figure 3 depicts this schematically.

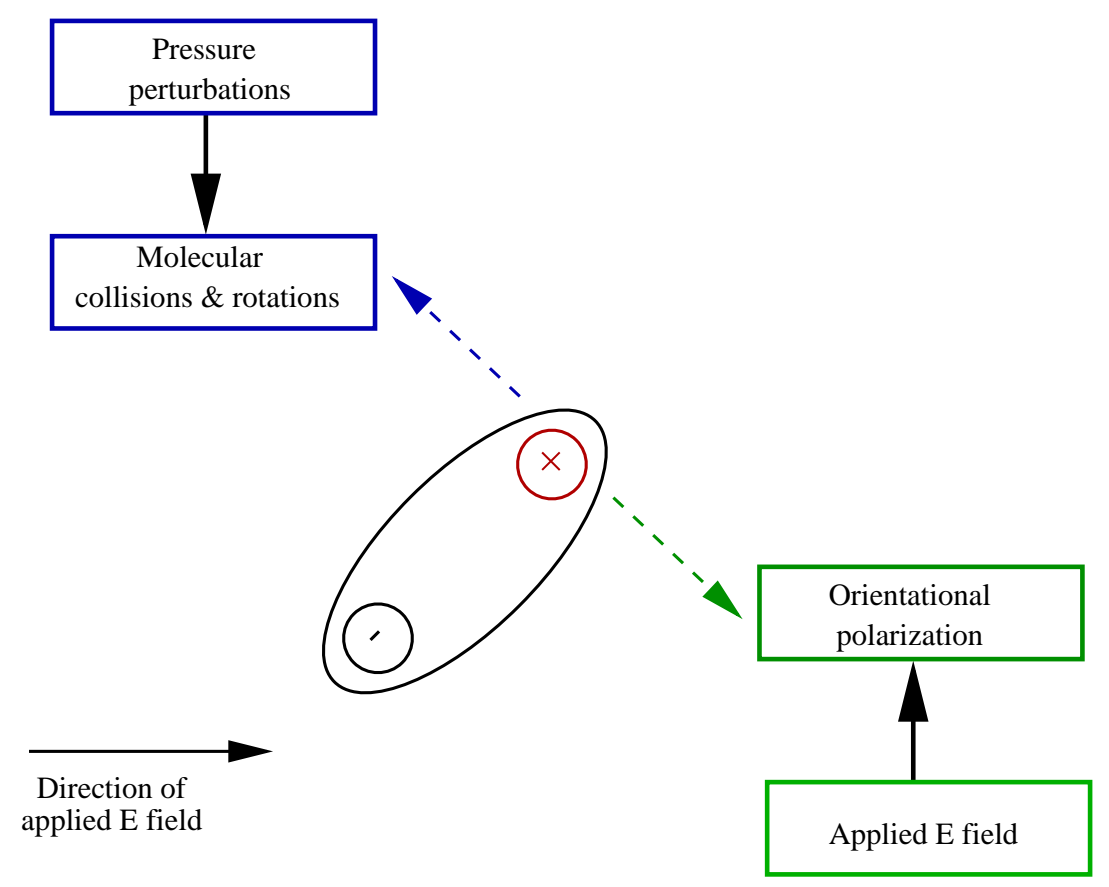

Figure 3: Pressure-dependence of orientational polarization

This interaction makes sense intuitively; however, we want to better understand the interaction mechanism. Specifically, we want to consider each polarization parameter individually and address its possible pressure-dependence. 
We begin with the static permittivity $\epsilon_{s}$, which is the electric permittivity of a dielectric in the presence of a constant applied electric field. In 1850 and 1879 respectively, Clausius and Mossitti independently discovered that for any given material, the quantity

$$
\left(\frac{\epsilon_{s}}{\epsilon_{0}}-1\right) /\left(\frac{\epsilon_{s}}{\epsilon_{0}}+2\right)
$$

is proportional to the material density (page 155, [18]; page 140, [25]). Pressure variations in the material cause changes in its density. These changes are reflected in the static permittivity of the material due to the law of Clausius and Mossitti. So the static permittivity can be expected to depend on pressure.

The pressure-dependence of $\epsilon_{s}$ does not necessarily suggest the pressure-dependence of $\epsilon_{\infty}$, the permittivity of a material under a very high frequency electric field. However in the interest of generality, we allow for the possibility that $\epsilon_{\infty}$ is pressure-dependent. Pressureindependent behavior of $\epsilon_{\infty}$ is just a special case of our model (see Section 2.1) with $\kappa_{\zeta}=0$ so that $\epsilon_{\infty}(p)=\zeta_{0}$.

Lastly we examine the feasibility of the pressure-dependence of the relaxation parameter $\tau$. To do so we consider a dipolar liquid which consists of freely moving molecules. If an individual dipole changes its orientation, the nearby dipoles shift to compensate and produce a new equilibrium position. Their collective motion can be viewed as a viscous frictional damping force that acts on the original dipole. When an electric field is applied, its force causes the dipole to align itself with the field. The rate of alignment depends on the amount of friction. However since the dipole is subject to the effects of Brownian motion, this rate also depends on thermal fluctuations. Taking this into account Debye derived the following expression for the relaxation (page $73,[2]$ )

$$
\tau=\frac{\xi}{2 k_{B} T}
$$

where $\xi$ is the frictional constant. Dipoles arranged in smaller groups are less apt to resist reorientation [13]. This leads to diminished frictional effects. Variations in pressure likely alter the cohesion of dipole groupings and thus affect the friction. A specific example of this relationship is given in (page 63, [21]) for hard sphere fluids. In this case, the frictional viscosity constant is given by

$$
\xi=\frac{k_{B} T}{m D}
$$

where $m$ is the particle mass and $D$ is the self-diffusion coefficient. The self-diffusion coefficient is pressure-dependent; that is 


$$
D=\frac{1}{2} R\left(\frac{\pi k_{B} T}{m}\right)^{\frac{1}{2}}\left(\frac{p}{\rho k_{B} T}-1\right)^{-1}
$$

where $R$ is the hard sphere diameter, $p$ is the pressure, and $\rho$ is the liquid density. Clearly in this example the relaxation parameter $\tau$ is pressure-dependent.

\section{Theoretical results}

In order to consider (4) from a theoretical perspective, it is convenient to write it in variational form. We formulate the Gelfand triple $V \hookrightarrow H \hookrightarrow V^{*}$, where $H=L^{2}(0,1)$ and $V=H_{R}^{1}(0,1) \equiv\left\{\phi \in H^{1}(0,1): \phi(1)=0\right\}$. We let $\left\langle\cdot, \cdot>\right.$ denote the usual $L^{2}$ inner product. Then we may write (4) as a variational form of Maxwell's equation in second order form

$$
\begin{gathered}
<a \ddot{E}(t), \phi>_{V^{*}, V}+<b \dot{E}(t), \phi>+<e \ddot{P}(t), \phi> \\
+c \dot{E}(t, 0) \phi(0)+\sigma_{1}(E(t), \phi)=<F(t), \phi>_{V^{*}, V}, \quad t \in[0, T],
\end{gathered}
$$

for all $\phi \in V$. The sesquilinear form $\sigma_{1}$ is defined by

$$
\sigma_{1}(\phi, \psi)=c^{2}<\phi^{\prime}, \psi^{\prime}>
$$

where $c^{2}=\frac{1}{\epsilon_{0} \mu_{0}}>0$ is constant and the parameter functions $a, b$, and $e$ are determined by the geometry, conductivity, and instantaneous polarization of the dielectric. Since the absorbing boundary condition $\dot{E}-c E^{\prime}=0$ at $z=0$ is a natural condition, we incorporate it into the variational formulation of the equation. The superconductive boundary condition at $z=1$ however is essential and is imposed in the definition of $V$.

Motivated by the polarization model described in Section 2, we may rewrite (9) as the general variational form

$$
\begin{gathered}
<a \ddot{E}(t), \phi>_{V^{*}, V}+<b \dot{E}(t), \phi>+<h E(t), \phi> \\
+<\int_{0}^{t} G(t, s, \cdot) E(s, \cdot) d s, \phi> \\
+c \dot{E}(t, 0) \phi(0)+\sigma_{1}(E(t), \phi)=<F(t), \phi>_{V^{*}, V}, \quad \phi \in V, \quad t \in[0, T] \\
E(0, z)=E_{0}(z) \quad \dot{E}(0, z)=E_{1}(z)
\end{gathered}
$$


where as in (9) the coefficients and kernel function are determined by system characteristics. For the pressure-dependent Debye polarization model given in Section 2, we have

$$
\begin{aligned}
a(t, z) & =1+\left(\epsilon_{\infty}-1\right) I_{\left(z_{1}, 1\right)}=1+\left(\zeta_{0}+\kappa_{\zeta} p(t, z)-1\right) I_{\left(z_{1}, 1\right)} \\
b(t, z) & =\left(\frac{\sigma}{\epsilon_{0}}+\frac{1}{\epsilon_{0}} \frac{\epsilon_{0}\left(\gamma_{0}-\zeta_{0}+\left(\kappa_{\gamma}-\kappa_{\zeta}\right) p(t, z)\right)}{\left(\tau_{0}+\kappa_{\tau} p(t, z)\right)}\right) I_{\left(z_{1}, 1\right)} \\
h(t, z) & =\frac{1}{\epsilon_{0}}\left(\frac{\epsilon_{0}\left(\kappa_{\gamma}-\kappa_{\zeta}\right) \dot{p}(t, z)}{\left(\tau_{0}+\kappa_{\tau} p(t, z)\right)}-\frac{\left(1+\kappa_{\tau} \dot{p}(t, z)\right) \epsilon_{0}\left(\gamma_{0}-\zeta_{0}+\left(\kappa_{\gamma}-\kappa_{\zeta}\right) p(t, z)\right)}{\left(\tau_{0}+\kappa_{\tau} p(t, z)\right)^{2}}\right) I_{\left(z_{1}, 1\right)} \\
G(t, s, z) & =\frac{1}{\epsilon_{0}} \frac{\left(1+\kappa_{\tau} \dot{p}(t, z)\right) \epsilon_{0}\left(\gamma_{0}-\zeta_{0}+\left(\kappa_{\gamma}-\kappa_{\zeta}\right) p(s, z)\right)}{\left(\tau_{0}+\kappa_{\tau} p(t, z)\right)^{2}\left(\tau_{0}+\kappa_{\tau} p(s, z)\right)} \exp \left(\int_{s}^{t} \frac{-d \xi}{\tau_{0}+\kappa_{\tau} p(\xi, z)}\right) I_{\left(z_{1}, 1\right)} \\
c^{2} & =\frac{1}{\epsilon_{0} \mu_{0}} \\
F(t, z) & =-\frac{1}{\epsilon_{0}} \dot{J}_{s}(t) \\
\sigma_{1}(\phi, \psi) & =c^{2}\left\langle\phi^{\prime}, \psi^{\prime}\right\rangle .
\end{aligned}
$$

(Here $I_{\Omega}$ is the indicator or characteristic function for a set $\Omega$.) We note that the sesquilinear form $\sigma_{1}: V \times V \rightarrow \mathbb{R}$ is $V$-continuous and $V$-elliptic, so that there exist positive constants $c_{1}, c_{2}$ such that the following inequalities hold.

$$
\begin{gathered}
\sigma_{1}(\phi, \psi)=c^{2}<\phi^{\prime}, \psi^{\prime}>\leq c^{2}\left|\phi^{\prime}\right|_{H}\left|\psi^{\prime}\right|_{H} \leq c_{1}|\phi|_{V}|\psi|_{V} \\
\sigma_{1}(\phi, \phi)=c^{2}<\phi^{\prime}, \phi^{\prime}>=c^{2}\left|\phi^{\prime}\right|_{H}^{2} \geq c_{2}|\phi|_{V}^{2}
\end{gathered}
$$

In [9], the well-posedness of (10) is considered for general coefficient, kernel, and forcing functions under the following assumptions

A1) The coefficient $a$ along with its derivatives $\dot{a}$ and $\ddot{a}$ are in $L^{\infty}\left(0, T ; L^{\infty}[0,1]\right)$, and for all $z \in[0,1], a(z) \geq a_{0}$, for some $1 \geq a_{0}>0$.

A2) The coefficient $b$ and its time derivative $\dot{b}$ are in $L^{\infty}\left(0, T ; L^{\infty}[0,1]\right)$ and $b(t, z) \geq 0$ for all $(t, z) \in[0, T] \times[0,1]$.

A3) The coefficient $h$ is in $L^{\infty}\left(0, T ; L^{\infty}[0,1]\right)$.

A4) The kernel function $G$ is in $L^{\infty}\left([0, T] \times[0, T] ; L^{\infty}[0,1]\right)$.

A5) The sequilinear form $\sigma_{1}$ is given by $\sigma_{1}(\phi, \psi)=c^{2}<\phi^{\prime}, \psi^{\prime}>$ for $\phi, \psi \in V$ with $c>0$. 
A6) The forcing function $F$ is in $H^{1}\left(0, T, V^{*}\right)$.

The result is the following theorem. (See [9] for a detailed proof.)

Theorem 1: Under assumptions A1)-A6), the system (10) possesses a unique solution and $(E, \dot{E})$ depends continuously on initial data $\left(E_{0}, E_{1}\right)$ and forcing function $F$ from $\left(E_{0}, E_{1}, F\right) \in V \times H \times H^{1}\left(0, T ; V^{*}\right)$ to $(E, \dot{E}) \in L^{2}(0, T ; V) \times L^{2}(0, T ; H)$.

We may verify that assumptions A1)-A6) hold for (11) - see [9].

\subsection{Estimation of parameters in the general variational form}

The previous well-posedness result provides a framework in which to formulate parameter estimation problems. As described in Section 1, the general Maxwell system treated by this result arises from a class electromagnetic interrogation problems. The crux of these problems is estimation of certain parameter values, namely dielectric constants and conductivity coefficients, for the material under interrogation. The estimation problem typically involves finding the parameter values that provide the best fit between the model and data collected from the actual system, using, for example, a least squares criterion. These parameter estimates may then be used to characterize the material.

In practice, the experimental data is compared with finite dimensional numerical approximations to the model. In this section, we examine the relationship between the parameter estimation problems for the original system (10) and for a corresponding finite dimensional system. We suppose that the coefficients and sesquilinear form in both (10) and its finite dimensional approximation depend on a parameter $q$ in a set $Q$. If the exact solution to the original system (10) were accessible, we would consider the problem of minimizing the least squares cost functional

$$
J(q, w)=\sum_{i=1}^{N_{t}}\left|\mathcal{O} E\left(t_{i} ; q\right)-w_{i}\right|^{2}
$$

over $q \in Q$ where $w=\left\{w_{i}\right\}_{i=1}^{N_{t}}$ is a set of observations taken at times $t_{i}, Q$ is a set of admissible parameters, and $\mathcal{O}$ is an observation operator. The form of $\mathcal{O}$ depends on the particular application and set of observations. For example, if $w_{i}$ is a measurement of the electric field taken at a spatial point $z$ at time $t_{i}$, then the operator $\mathcal{O}$ entails evaluations of the function $E\left(t_{i}, \cdot ; q\right)$ at a point in space. Since we cannot obtain a closed form solution to $(10)$, we use the solution $E^{N}(t ; q)$ to a finite dimensional approximating system. The solution $E^{N}(t ; q)$ lies in $V^{N}$, a finite dimesional subset of $V$, and satisfies 


$$
\begin{gathered}
<a(q) \ddot{E}^{N}(t), \phi>_{V^{*}, V}+<b(q) \dot{E}^{N}(t), \phi>+<h(q) E^{N}(t), \phi> \\
+<\int_{0}^{t} G(t, s, \cdot ; q) E^{N}(s, \cdot) d s, \phi> \\
+c \dot{E}^{N}(t, 0) \phi(0)+\sigma_{1}(q)\left(E^{N}(t), \phi\right)=<F(t), \phi>_{V^{*}, V} \\
E^{N}(0, z)=\mathcal{P}^{N} E_{0}(z) \quad \dot{E}^{N}(0, z)=\mathcal{P}^{N} E_{1}(z)
\end{gathered}
$$

for all $\phi \in V^{N}$. In particular, we define the piecewise linear basis elements $\left\{\phi_{j}^{N}\right\}_{j=0}^{N-1}$ with nodal values $\phi_{j}^{N}(k / N)=\delta_{k j}, k=0,1, \ldots, N$, and let $V^{N}=\operatorname{span}\left\{\phi_{0}^{N}, \phi_{1}^{N}, \ldots, \phi_{N-1}^{N}\right\} \subset V$. Then we define $\mathcal{P}^{N}$ to be the quasi- $L^{2}(0,1)$ projection (see [23], [4], [12]) of $V^{*}$ onto $V^{N}$ defined by

$$
<\mathcal{P}^{N} v^{*}, v^{N}>_{N}=<v^{*}, v^{N}>_{V^{*}, V} \quad \text { for } v^{*} \in V^{*} \text { and for all } v^{N} \in V^{N}
$$

where

$$
<w^{N}, v^{N}>_{N} \equiv \int_{0}^{1} I^{N}\left(w^{N} v^{N}\right)(z) d z
$$

and $I^{N}$ is the nodal value linear interpolation operator for $V^{N}$. It is shown in [23] that the operator $\mathcal{P}^{N}$ is well-defined and satisfies

$$
\begin{aligned}
\left|\mathcal{P}^{N} \phi\right|_{H} & \leq K_{1}^{\prime}|\phi|_{H} \text { for } \phi \in H \\
\left|\mathcal{P}^{N} \phi\right|_{V} & \leq K_{2}^{\prime}|\phi|_{V} \text { for } \phi \in V .
\end{aligned}
$$

As expected, the corresponding cost functional for the finite dimensional system is

$$
J^{N}(q, w)=\sum_{i=1}^{N_{t}}\left|\mathcal{O} E^{N}\left(t_{i} ; q\right)-w_{i}\right|^{2}
$$

Again the form of the operator $\mathcal{O}$ is chosen to correspond to the type of data collected.

In [9], we established the well-posedness of (10) with solutions $E$ in $L^{2}(0, T ; V)$ and $\dot{E} \in$ $L^{2}(0, T ; H)$, where $V=H_{R}^{1}(0,1)$ and $H=L^{2}(0,1)$, and we also verified that a unique solution to (13) exists. These results hold provided that, for each $q \in Q$, Assumptions A1)A6) are satisfied and the sesquilinear form $\sigma_{1}$ is $V$-continuous and $V$-elliptic. Moreover in [24], we show that the solution $E$ of (10) has the enhanced regularity $E \in H^{3}\left(0, T ; V^{*}\right) \cap$ 
$H^{2}(0, T ; H) \cap H^{1}(0, T ; V)$ under consistency conditions for the initial conditions and the following assumptions:

A7) The second time derivative, $\ddot{b}$, of $b$ is in $L^{\infty}\left(0, T ; L^{\infty}[0,1]\right)$.

A8) The first and second time derivatives, $\dot{h}$ and $\ddot{h}$, of $h$ are in $L^{\infty}\left(0, T ; L^{\infty}[0,1]\right)$.

A9) The first and second derivatives with respect to the first temporal variable, $\frac{d}{d t} G$ and $\frac{d^{2}}{d t^{2}} G$, of the kernel function $G$ are in $L^{\infty}\left((0, T) \times(0, T) ; L^{\infty}[0,1]\right)$.

A10) The forcing function $F$ is in $H^{2}\left(0, T, V^{*}\right)$ and is of the form $F(t, z)=\tilde{g}(t) \delta(z)$ with $\tilde{g}(t) \in H^{2}(0, T)$ and $\tilde{g}(0)=\dot{\tilde{g}}(0)=0$. (This assumption replaces A6).)

(Verification of these assumptions A7)-A9) for the pressure-dependent Debye polarization model are also given in [24].)

We now make the following assumptions about the set of admissible parameters $Q$, the state space $V^{N}$, and the projection operator $\mathcal{P}^{N}$.

B1) The finite dimensional set $Q$ lies in a metric space $\tilde{Q}$ with a metric $\tilde{d}$ and is compact with respect to this metric.

B2) The finite dimensional subspaces $V^{N}$ are subsets of $V$.

B3) For each $\phi \in V,\left|\phi-\mathcal{P}^{N} \phi\right|_{V} \rightarrow 0$ as $N \rightarrow \infty$.

B4) For each $\phi \in H,\left|\phi-\mathcal{P}^{N} \phi\right|_{H} \rightarrow 0$ as $N \rightarrow \infty$.

Verifications of B3) and B4) for our particular $\mathcal{P}^{N}$ are given in [23]. We now make a further assumption on the sesquilinear form $\sigma_{1}$. We assume that $\sigma_{1}=\sigma_{1}(q)$ is defined on $Q$ and satisfies

H1)

$$
\left|\sigma_{1}\left(q_{1}\right)(\phi, \psi)-\sigma_{1}\left(q_{2}\right)(\phi, \psi)\right| \leq \gamma \tilde{d}\left(q_{1}, q_{2}\right)|\phi|_{V}|\psi|_{V}
$$

for $q_{1}, q_{2} \in Q$ where $\gamma$ depends only on $Q$.

For the electromagnetic system in consideration in this paper, Assumption H1) is unnecessary since $\sigma_{1}$ is independent of $q$. However for the purpose of establishing a more general result, we do not assume here that our sesquilinear form is parameter independent.

Furthermore we make the following assumption about our coefficients.

A11) The coefficients depend continuously on $q$ so that as $\tilde{d}\left(q, q^{N}\right) \rightarrow 0$, we have 


$$
\begin{array}{ll}
\text { i) } & \left|a(q)-a\left(q^{N}\right)\right|_{L^{\infty}} \rightarrow 0 \\
\text { ii) } & \left|b(q)-b\left(q^{N}\right)\right|_{L^{\infty}} \rightarrow 0 \\
\text { iii) } & \left|h(q)-h\left(q^{N}\right)\right|_{L^{\infty}} \rightarrow 0 \\
\text { iv }) & \left|G(q)-G\left(q^{N}\right)\right|_{L^{\infty}} \rightarrow 0 .
\end{array}
$$

The above continuity along with the compactness of $Q$ implies that the images $a(Q), b(Q)$, $h(Q)$, and $G(Q)$ are compact. Thus each coefficient can be bounded independently of $q$. We assume throughout that all bounds on our coefficients do not depend on $q$.

By solving the parameter estimation problems related to (13), (15) we obtain a sequence of estimates $\left\{\bar{q}^{N}\right\}$. We wish to demonstrate that under certain conditions this sequence (or a subsequence) converges to the estimate corresponding to the problem related to (10), (12). In order to do this, we state the following claim, which can be found (along with a proof) as Theorem 5.1 in [11].

Theorem 2: To obtain convergence of at least a subsequence of $\left\{\bar{q}^{N}\right\}$ to a solution $\bar{q}$ of minimizing (12) subject to (10), it suffices, under assumption B1), to argue that for arbitrary sequences $\left\{q^{N}\right\}$ in $Q$ with $q^{N} \rightarrow q$ in $Q$, we have

$$
\mathcal{O} E^{N}\left(t ; q^{N}\right) \rightarrow \mathcal{O} E(t ; q) .
$$

In [11], the operator $\mathcal{O}$ is general enough to include functions that map functions $f$ such that $f: \mathcal{T} \rightarrow \mathcal{V}$ to the space of observations $\mathcal{W}$, where $\mathcal{T}$ is an appropriately chosen (see [11] and [8]) subset of $[0, T]$ that contains the times of observation and $\mathcal{V}$ is a space containing $E(t, \cdot)$. In the numerical examples presented in this paper, the observations correspond to the values of the electric field at the point $z=0$ at various times, i.e., $\left\{E\left(t_{i}, 0\right)\right\}$; thus the operator $\mathcal{O}$ involves pointwise evaluation of $E$ at many points in time and one specific point in space.

We suppose that $V^{N}$ and $\mathcal{P}^{N}$ satisfy B2)-B4), the sesquilinear form $\sigma_{1}$ satisfies H1), the coefficients satisfy assumptions A1)-A11) and we let $q^{N} \in Q$ be arbitary such that $q^{N} \rightarrow q$ in $Q$. Our primary goal is to show that as $N \rightarrow \infty$

$$
E^{N}\left(t, 0 ; q^{N}\right) \rightarrow E(t, 0 ; q)
$$

for each $t \in[0, T]$. However, here we verify a more general result. We show that for each $t \in[0, T]$ 


$$
\begin{aligned}
& E^{N}\left(t ; q^{N}\right) \rightarrow E(t ; q) \text { in the } V \text { norm } \\
& \dot{E}^{N}\left(t ; q^{N}\right) \rightarrow \dot{E}(t ; q) \text { in the } H \text { norm }
\end{aligned}
$$

as $N \rightarrow \infty$, where $E^{N}, \dot{E}^{N}$ are the solutions to (13) and $E, \dot{E}$ are the solutions to (10). We note that we may evaluate these functions pointwise in $t$ due to the enhanced regularity of solutions. Moveover, using the equivalence of norms, we see that (17) implies (16) and we have the result we need for our computations.

We point out that for a sequence $q^{N}=q$ for all $N$, the desired result implies convergence of the finite dimensional approximation to the true solution. This is important when considering numerical approximations to the solution.

We have established previously that the solution of (10) satisfies $E(t) \in V$ and $\dot{E}(t) \in H$ for each $t$. Since

$$
\left|E^{N}\left(t ; q^{N}\right)-E(t ; q)\right|_{V} \leq\left|E^{N}\left(t ; q^{N}\right)-\mathcal{P}^{N} E(t ; q)\right|_{V}+\left|\mathcal{P}^{N} E(t ; q)-E(t ; q)\right|_{V}
$$

and B3) guarantees $\left|\mathcal{P}^{N} E(t ; q)-E(t ; q)\right|_{V} \rightarrow 0$ as $N \rightarrow \infty$, we need only show that

$$
\left|E^{N}\left(t ; q^{N}\right)-\mathcal{P}^{N} E(t ; q)\right|_{V} \rightarrow 0 \text { as } N \rightarrow \infty
$$

for each $t \in[0, T]$. In the same way, it suffices to show that

$$
\left|\dot{E}^{N}\left(t ; q^{N}\right)-\mathcal{P}^{N} \dot{E}(t ; q)\right|_{H} \rightarrow 0 \text { as } N \rightarrow \infty
$$

for each $t \in[0, T]$ to obtain the second result.

We let $E^{N}=E^{N}\left(t ; q^{N}\right), E=E(t ; q)$, and $\Delta^{N} \equiv E^{N}\left(t ; q^{N}\right)-\mathcal{P}^{N} E(t ; q)$.

Subtracting (10) from (13), we have for $\phi \in V^{N}$

$$
\begin{gathered}
<a\left(q^{N}\right) \ddot{E}^{N}-a(q) \ddot{E}, \phi>+<b\left(q^{N}\right) \dot{E}^{N}-b(q) \dot{E}, \phi> \\
+<h\left(q^{N}\right) E^{N}-h(q) E, \phi> \\
+<\int_{0}^{t}\left(G\left(q^{N}\right) E^{N}-G(q) E\right) d s, \phi>+c\left(\dot{E}^{N}(t, 0)-\dot{E}(t, 0)\right) \phi(0) \\
+\sigma_{1}\left(q^{N}\right)\left(E^{N}, \phi\right)-\sigma_{1}(q)(E, \phi)=0 .
\end{gathered}
$$


We add and subtract $\mathcal{P}^{N} E$ and its derivatives and rearrange terms to obtain

$$
\begin{aligned}
& <a\left(q^{N}\right)\left(\ddot{E}^{N}-\mathcal{P}^{N} \ddot{E}\right), \phi>+\sigma_{1}\left(q^{N}\right)\left(E^{N}-\mathcal{P}^{N} E, \phi\right) \\
& +c\left(\dot{E}^{N}(t, 0)-\mathcal{P}^{N} \dot{E}(t, 0)\right) \phi(0) \\
& =<a(q) \ddot{E}, \phi>-<a\left(q^{N}\right) \mathcal{P}^{N} \ddot{E}, \phi>+\sigma_{1}(q)(E, \phi)-\sigma_{1}\left(q^{N}\right)\left(\mathcal{P}^{N} E, \phi\right) \\
& +c\left(\dot{E}(t, 0)-\mathcal{P}^{N} \dot{E}(t, 0)\right) \phi(0)+<b(q) \dot{E}-b\left(q^{N}\right) \dot{E}^{N}, \phi> \\
& +<h(q) E-h\left(q^{N}\right) E^{N}, \phi>+<\int_{0}^{t} G(q) E-G\left(q^{N}\right) E^{N} d s, \phi>.
\end{aligned}
$$

We choose the test function $\phi=\dot{\Delta}^{N} \in V^{N}$ so that

$$
\begin{aligned}
& <a\left(q^{N}\right)\left(\ddot{E}^{N}-\mathcal{P}^{N} \ddot{E}\right), \dot{\Delta}^{N}>+\sigma_{1}\left(q^{N}\right)\left(E^{N}-\mathcal{P}^{N} E, \dot{\Delta}^{N}\right) \\
& +c\left(\dot{E}^{N}(t, 0)-\mathcal{P}^{N} \dot{E}(t, 0)\right) \dot{\Delta}^{N}(t, 0)= \\
& <a(q) \ddot{E}, \dot{\Delta}^{N}>-<a\left(q^{N}\right) \mathcal{P}^{N} \ddot{E}, \dot{\Delta}^{N}>+\sigma_{1}(q)\left(E, \dot{\Delta}^{N}\right)-\sigma_{1}\left(q^{N}\right)\left(\mathcal{P}^{N} E, \dot{\Delta}^{N}\right) \\
& +c\left(\dot{E}(t, 0)-\mathcal{P}^{N} \dot{E}(t, 0)\right) \dot{\Delta}^{N}(t, 0)+<b(q) \dot{E}-b\left(q^{N}\right) \dot{E}^{N}, \dot{\Delta}^{N}> \\
& +<h(q) E-h\left(q^{N}\right) E^{N}, \dot{\Delta}^{N}>+<\int_{0}^{t}\left(G(q) E-G\left(q^{N}\right) E^{N}\right) d s, \dot{\Delta}^{N}>.
\end{aligned}
$$

We note that

$$
\begin{aligned}
& 2<a\left(q^{N}\right)\left(\ddot{E}^{N}-\mathcal{P}^{N} \ddot{E}\right), \dot{\Delta}^{N}>+2 \sigma_{1}\left(q^{N}\right)\left(E^{N}-\mathcal{P}^{N} E, \dot{\Delta}^{N}\right) \\
& =\frac{d}{d t}\left(<a\left(q^{N}\right) \dot{\Delta}^{N}, \dot{\Delta}^{N}>+\sigma_{1}\left(q^{N}\right)\left(\Delta^{N}, \Delta^{N}\right)\right)-<\dot{a}\left(q^{N}\right) \dot{\Delta}^{N}, \dot{\Delta}^{N}>.
\end{aligned}
$$

Then we have 


$$
\begin{gathered}
\frac{1}{2} \frac{d}{d t}\left(<a\left(q^{N}\right) \dot{\Delta}^{N}, \dot{\Delta}^{N}>+\sigma_{1}\left(q^{N}\right)\left(\Delta^{N}, \Delta^{N}\right)\right)+c\left(\dot{\Delta}^{N}(t, 0)\right)^{2}= \\
<a(q) \ddot{E}, \dot{\Delta}^{N}>-<a\left(q^{N}\right) \mathcal{P}^{N} \ddot{E}, \dot{\Delta}^{N}>+<\dot{a}\left(q^{N}\right) \dot{\Delta}^{N}, \dot{\Delta}^{N}> \\
+\sigma_{1}(q)\left(E, \dot{\Delta}^{N}\right)-\sigma_{1}\left(q^{N}\right)\left(\mathcal{P}^{N} E, \dot{\Delta}^{N}\right) \\
+c\left(\dot{E}(t, 0)-\mathcal{P}^{N} \dot{E}(t, 0)\right) \dot{\Delta}^{N}(t, 0)+<b(q) \dot{E}-b\left(q^{N}\right) \dot{E}^{N}, \dot{\Delta}^{N}> \\
+<h(q) E-h\left(q^{N}\right) E^{N}, \dot{\Delta}^{N}>+<\int_{0}^{t}\left(G(q) E-G\left(q^{N}\right) E^{N}\right) d s, \dot{\Delta}^{N}>.
\end{gathered}
$$

Integration with respect to $t$ yields

$$
\begin{gathered}
<a\left(q^{N}\right) \dot{\Delta}^{N}(t), \dot{\Delta}^{N}(t)>+\sigma_{1}\left(q^{N}\right)\left(\Delta^{N}(t), \Delta^{N}(t)\right) \\
+2 \int_{0}^{t} c\left(\dot{\Delta}^{N}(\xi, 0)\right)^{2} d \xi= \\
2 \int_{0}^{t}\left\{<a(q) \ddot{E}, \dot{\Delta}^{N}>-<a\left(q^{N}\right) \mathcal{P}^{N} \ddot{E}, \dot{\Delta}^{N}>+<\dot{a}\left(q^{N}\right) \dot{\Delta}^{N}, \dot{\Delta}^{N}>\right. \\
+\sigma_{1}(q)\left(E, \dot{\Delta}^{N}\right)-\sigma_{1}\left(q^{N}\right)\left(\mathcal{P}^{N} E, \dot{\Delta}^{N}\right) \\
+c\left(\dot{E}(\xi, 0)-\mathcal{P}^{N} \dot{E}(\xi, 0)\right) \dot{\Delta}^{N}(\xi, 0)+<b(q) \dot{E}-b\left(q^{N}\right) \dot{E}^{N}, \dot{\Delta}^{N}> \\
\left.+<h(q) E-h\left(q^{N}\right) E^{N}, \dot{\Delta}^{N}>+<\int_{0}^{\xi} G(q) E-G\left(q^{N}\right) E^{N} d s, \dot{\Delta}^{N}>\right\} d \xi \\
+<a\left(q^{N}\right) \dot{\Delta}^{N}(0), \dot{\Delta}^{N}(0)>+\sigma_{1}\left(q^{N}\right)\left(\Delta^{N}(0), \Delta^{N}(0)\right) .
\end{gathered}
$$

We now use the definition of $\Delta^{N}$ to obtain

$$
\begin{aligned}
& \Delta^{N}(0)=E^{N}(0)-\mathcal{P}^{N} E(0)=E^{N}(0)-\mathcal{P}^{N} E_{0}=0 \\
& \dot{\Delta}^{N}(0)=\dot{E}^{N}(0)-\mathcal{P}^{N} \dot{E}(0)=\dot{E}^{N}(0)-\mathcal{P}^{N} E_{1}=0 .
\end{aligned}
$$

We may then write 


$$
\begin{gathered}
<a\left(q^{N}\right) \dot{\Delta}^{N}(t), \dot{\Delta}^{N}(t)>+\sigma_{1}\left(q^{N}\right)\left(\Delta^{N}(t), \Delta^{N}(t)\right) \\
+2 \int_{0}^{t} c\left(\dot{\Delta}^{N}(\xi, 0)\right)^{2} d \xi= \\
2 \int_{0}^{t}\left\{<a(q) \ddot{E}, \dot{\Delta}^{N}>-<a\left(q^{N}\right) \mathcal{P}^{N} \ddot{E}, \dot{\Delta}^{N}>+<\dot{a}\left(q^{N}\right) \dot{\Delta}^{N}, \dot{\Delta}^{N}>\right. \\
+\sigma_{1}(q)\left(E, \dot{\Delta}^{N}\right)-\sigma_{1}\left(q^{N}\right)\left(\mathcal{P}^{N} E, \dot{\Delta}^{N}\right) \\
+c\left(\dot{E}(\xi, 0)-\mathcal{P}^{N} \dot{E}(\xi, 0)\right) \dot{\Delta}^{N}(\xi, 0)+<b(q) \dot{E}-b\left(q^{N}\right) \dot{E}^{N}, \dot{\Delta}^{N}> \\
\left.+<h(q) E-h\left(q^{N}\right) E^{N}, \dot{\Delta}^{N}>+<\int_{0}^{\xi} G(q) E-G\left(q^{N}\right) E^{N} d s, \dot{\Delta}^{N}>\right\} d \xi .
\end{gathered}
$$

In order to bound the right side of (18), we derive the following estimates:

Estimate 1:

$$
\begin{aligned}
& \int_{0}^{t}\left(2<a(q) \ddot{E}, \dot{\Delta}^{N}>-2<a\left(q^{N}\right) \mathcal{P}^{N} \ddot{E}, \dot{\Delta}^{N}>+<\dot{a}\left(q^{N}\right) \dot{\Delta}^{N}, \dot{\Delta}^{N}>\right) d \xi \\
= & \int_{0}^{t}\left(2<\left(a(q)-a\left(q^{N}\right)\right) \ddot{E}, \dot{\Delta}^{N}>+2<a\left(q^{N}\right)\left(\ddot{E}-\mathcal{P}^{N} \ddot{E}\right), \dot{\Delta}^{N}>\right. \\
& \left.+<\dot{a}\left(q^{N}\right) \dot{\Delta}^{N}, \dot{\Delta}^{N}>\right) d \xi \\
\leq & \int_{0}^{t}\left|\left(a(q)-a\left(q^{N}\right)\right) \ddot{E}\right|_{H}^{2}+\left|a\left(q^{N}\right)\left(\ddot{E}-\mathcal{P}^{N} \ddot{E}\right)\right|_{H}^{2}+\frac{1}{2}\left(5+\left|\dot{a}\left(q^{N}\right)\right|_{H}^{2}\right)\left|\dot{\Delta}^{N}\right|_{H}^{2} d \xi \\
\leq & \left|a(q)-a\left(q^{N}\right)\right|_{L^{\infty}}^{2} \int_{0}^{t}|\ddot{E}|_{H}^{2} d \xi+\left|a\left(q^{N}\right)\right|_{L^{\infty}}^{2} \int_{0}^{t}\left|\ddot{E}-\mathcal{P}^{N} \ddot{E}\right|_{H}^{2} d \xi \\
& +\frac{1}{2}\left(5+\left|\dot{a}\left(q^{N}\right)\right|_{L^{\infty}}^{2}\right) \int_{0}^{t}\left|\dot{\Delta}^{N}\right|_{H}^{2} d \xi .
\end{aligned}
$$

Estimate 2:

$$
\begin{aligned}
& 2 \int_{0}^{t} \sigma_{1}(q)\left(E, \dot{\Delta}^{N}\right)-\sigma_{1}\left(q^{N}\right)\left(\mathcal{P}^{N} E, \dot{\Delta}^{N}\right) d \xi \\
= & 2 \int_{0}^{t} \sigma_{1}\left(q^{N}\right)\left(\mathcal{P}^{N} \dot{E}, \Delta^{N}\right)-\sigma_{1}(q)\left(\dot{E}, \Delta^{N}\right) d \xi+2\left(\sigma_{1}(q)\left(E(t), \Delta^{N}(t)\right)\right. \\
& \left.-\sigma_{1}\left(q^{N}\right)\left(\mathcal{P}^{N} E(t), \Delta^{N}(t)\right)\right)
\end{aligned}
$$




$$
\begin{aligned}
= & 2 \int_{0}^{t} \sigma_{1}\left(q^{N}\right)\left(\mathcal{P}^{N} \dot{E}-\dot{E}, \Delta^{N}\right)+\sigma_{1}\left(q^{N}\right)\left(\dot{E}, \Delta^{N}\right)-\sigma_{1}(q)\left(\dot{E}, \Delta^{N}\right) d \xi \\
& +2\left(\sigma_{1}(q)\left(E(t), \Delta^{N}(t)\right)-\sigma_{1}\left(q^{N}\right)\left(E(t), \Delta^{N}(t)\right)\right. \\
& \left.+\sigma_{1}\left(q^{N}\right)\left(E(t)-\mathcal{P}^{N} E(t), \Delta^{N}(t)\right)\right) \\
\leq & \int_{0}^{t} c_{1}^{2}\left|\mathcal{P}^{N} \dot{E}-\dot{E}\right|_{V}^{2}+\gamma^{2}\left(\tilde{d}\left(q, q^{N}\right)\right)^{2}|\dot{E}|_{V}^{2}+2\left|\Delta^{N}\right|_{V}^{2} d \xi \\
& +\frac{c_{1}^{2}}{\epsilon}\left|\mathcal{P}^{N} E(t)-E(t)\right|_{V}^{2}+\frac{\gamma^{2}}{\epsilon}\left(\tilde{d}\left(q, q^{N}\right)\right)^{2}|E(t)|_{V}^{2}+2 \epsilon\left|\Delta^{N}(t)\right|_{V}^{2}
\end{aligned}
$$

where $\epsilon>0$ is arbitrary.

\section{Estimate 3:}

$$
\begin{aligned}
& 2 c\left(\dot{E}(\xi, 0)-\mathcal{P}^{N} \dot{E}(\xi, 0)\right) \dot{\Delta}^{N}(\xi, 0) \\
\leq & c^{2}\left|\dot{E}(\xi, 0)-\mathcal{P}^{N} \dot{E}(\xi, 0)\right|^{2}+\left|\dot{\Delta}^{N}(\xi, 0)\right|^{2} \\
\leq & c^{2} K_{1}\left|\dot{E}-\mathcal{P}^{N} \dot{E}\right|_{V}^{2}+\left|\dot{\Delta}^{N}(\xi, 0)\right|^{2} .
\end{aligned}
$$

(Here we use the fact that $|\phi|_{V}^{2}$ is equivalent to $\left|\phi^{\prime}\right|_{H}^{2}+|\phi(0)|^{2}$ so that $|\phi|_{V}^{2} \geq \tilde{K}\left(\left|\phi^{\prime}\right|_{H}^{2}+\right.$ $\left.|\phi(0)|^{2}\right) \geq \tilde{K}|\phi(0)|^{2}$.)

Estimate 4:

$$
\begin{aligned}
& 2 \int_{0}^{t}<b(q) \dot{E}-b\left(q^{N}\right) \dot{E}^{N}, \dot{\Delta}^{N}>d \xi \\
= & 2 \int_{0}^{t}\left\{<b(q)\left(\dot{E}-\mathcal{P}^{N} \dot{E}\right), \dot{\Delta}^{N}>+<\left(b(q)-b\left(q^{N}\right)\right) \mathcal{P}^{N} \dot{E}, \dot{\Delta}^{N}>\right. \\
& \left.+<b\left(q^{N}\right) \dot{\Delta} \dot{\Delta}^{N}, \dot{\Delta}^{N}>\right\} d \xi \\
\leq & \int_{0}^{t}\left|b(q)\left(\dot{E}-\mathcal{P}^{N} \dot{E}\right)\right|_{H}^{2}+\left|b\left(q^{N}\right) \dot{\Delta}^{N}\right|_{H}^{2}+\left|\left(b(q)-b\left(q^{N}\right)\right) \mathcal{P}^{N} \dot{E}\right|_{H}^{2}+3\left|\dot{\Delta}^{N}\right|_{H}^{2} d \xi \\
\leq & |b(q)|_{L^{\infty}}^{2} \int_{0}^{t}\left|\dot{E}-\mathcal{P}^{N} \dot{E}\right|_{H}^{2} d \xi+\left(\left|b\left(q^{N}\right)\right|_{L^{\infty}}^{2}+3\right) \int_{0}^{t}\left|\dot{\Delta}^{N}\right|_{H}^{2} d \xi \\
& +\left|b(q)-b\left(q^{N}\right)\right|_{L^{\infty}}^{2} \int_{0}^{t}\left|\mathcal{P}^{N} \dot{E}\right|_{H}^{2} d \xi .
\end{aligned}
$$


Estimate 5:

$$
\begin{aligned}
& 2 \int_{0}^{t}<h(q) E-h\left(q^{N}\right) E^{N}, \dot{\Delta}^{N}>d \xi \\
= & 2 \int_{0}^{t}\left\{<h(q)\left(E-\mathcal{P}^{N} E\right), \dot{\Delta}^{N}>+<\left(h(q)-h\left(q^{N}\right)\right) \mathcal{P}^{N} E, \dot{\Delta}^{N}>\right. \\
& \left.+<h\left(q^{N}\right) \Delta^{N}, \dot{\Delta}^{N}>\right\} d \xi \\
\leq & \int_{0}^{t}\left(\left|h(q)\left(E-\mathcal{P}^{N} E\right)\right|_{H}^{2}+\left|h\left(q^{N}\right) \Delta^{N}\right|_{H}^{2}+3\left|\dot{\Delta}^{N}\right|_{H}^{2}\right. \\
& \left.+\left|\left(h(q)-h\left(q^{N}\right)\right) \mathcal{P}^{N} E\right|_{H}^{2}\right) d \xi \\
\leq & |h(q)|_{L^{\infty}}^{2} \int_{0}^{t}\left|E-\mathcal{P}^{N} E\right|_{H}^{2} d \xi+\left|h\left(q^{N}\right)\right|_{L^{\infty}}^{2} \int_{0}^{t}\left|\Delta^{N}\right|_{H}^{2} d \xi+3 \int_{0}^{t}\left|\dot{\Delta}^{N}\right|_{H}^{2} d \xi \\
& +\left|h(q)-h\left(q^{N}\right)\right|_{L^{\infty}}^{2} \int_{0}^{t}\left|\mathcal{P}^{N} E\right|_{H}^{2} d \xi .
\end{aligned}
$$

Estimate 6:

$$
\begin{aligned}
& 2 \int_{0}^{t}<\int_{0}^{\xi}\left(G(q) E-G\left(q^{N}\right) E^{N}\right) d s, \dot{\Delta}^{N}>d \xi \\
= & 2 \int_{0}^{t}<\int_{0}^{\xi} G(q)\left(E-\mathcal{P}^{N} E\right) d s, \dot{\Delta}^{N}>+<\int_{0}^{\xi}\left(G(q)-G\left(q^{N}\right)\right) \mathcal{P}^{N} E d s, \dot{\Delta}^{N}> \\
& +<\int_{0}^{\xi} G\left(q^{N}\right) \Delta^{N} d s, \dot{\Delta}^{N}>d \xi \\
\leq & \int_{0}^{t}\left\{\left|\int_{0}^{\xi} G(q)\left(E-\mathcal{P}^{N} E\right) d s\right|_{H}^{2}+\left|\int_{0}^{\xi}\left(G(q)-G\left(q^{N}\right)\right) \mathcal{P}^{N} E d s\right|_{H}^{2}\right. \\
& \left.+\left|\int_{0}^{\xi} G\left(q^{N}\right) \Delta^{N} d s\right|_{H}^{2}+3\left|\dot{\Delta}^{N}\right|_{H}^{2}\right\} d \xi \\
= & \int_{0}^{t} \int_{0}^{1}\left|\int_{0}^{\xi} G(q)\left(E-\mathcal{P}^{N} E\right) d s\right|^{2} d z d \xi \\
& +\int_{0}^{t} \int_{0}^{1}\left|\int_{0}^{\xi}\left(G(q)-G\left(q^{N}\right)\right) \mathcal{P}^{N} E d s\right|^{2} d z d \xi \\
& +\int_{0}^{t} \int_{0}^{1}\left|\int_{0}^{\xi} G\left(q^{N}\right) \Delta^{N} d s\right|^{2} d z d \xi+3 \int_{0}^{t}\left|\dot{\Delta}^{N}\right|_{H}^{2} d \xi \\
\leq & |G(q)|_{L^{\infty}}^{2} \int_{0}^{t} \int_{0}^{1}\left|\int_{0}^{\xi}\left(E-\mathcal{P}^{N} E\right) d s\right|^{2} d z d \xi+\left|G(q)-G\left(q^{N}\right)\right|_{L^{\infty}}^{2} \int_{0}^{t} \int_{0}^{1}\left|\int_{0}^{\xi} \mathcal{P}^{N} E d s\right|^{2} d z d \xi \\
& +\left|G\left(q^{N}\right)\right|_{L^{\infty}}^{2} \int_{0}^{t} \int_{0}^{1}\left|\int_{0}^{\xi} \Delta^{N} d s\right|^{2} d z d \xi+3 \int_{0}^{t}\left|\dot{\Delta}^{N}\right|_{H}^{2} d \xi
\end{aligned}
$$




$$
\begin{aligned}
\leq & |G(q)|_{L^{\infty}}^{2} T \int_{0}^{t} \int_{0}^{1}\left|E-\mathcal{P}^{N} E\right|_{L^{2}(0, \xi)}^{2} d z d \xi+\left|G(q)-G\left(q^{N}\right)\right|_{L^{\infty}}^{2} T \int_{0}^{t} \int_{0}^{1}\left|\mathcal{P}^{N} E\right|_{L^{2}(0, \xi)}^{2} d z d \xi \\
& +\left|G\left(q^{N}\right)\right|_{L^{\infty}}^{2} T \int_{0}^{t} \int_{0}^{1}\left|\Delta^{N}\right|_{L^{2}(0, \xi)}^{2} d z d \xi+3 \int_{0}^{t}\left|\dot{\Delta}^{N}\right|_{H}^{2} d \xi \\
\leq & |G(q)|_{L^{\infty}}^{2} T^{2} \int_{0}^{t}\left|E-\mathcal{P}^{N} E\right|_{H}^{2} d \xi+\left|G(q)-G\left(q^{N}\right)\right|_{L^{\infty}}^{2} T^{2} \int_{0}^{t}\left|\mathcal{P}^{N} E\right|_{H}^{2} d \xi \\
& +\left|G\left(q^{N}\right)\right|_{L^{\infty}}^{2} T^{2} \int_{0}^{t}\left|\Delta^{N}\right|_{H}^{2} d \xi+3 \int_{0}^{t}\left|\dot{\Delta^{N}}\right|_{H}^{2} d \xi .
\end{aligned}
$$

Using these estimates, Assumption H1), the $V$-continuity and $V$-ellipticity of $\sigma_{1}$, and the fact that $|\phi|_{H}^{2} \leq|\phi|_{V}^{2}$, we may rewrite (18) as

$$
\begin{aligned}
& \left|\sqrt{a\left(q^{N}\right)} \dot{\Delta}^{N}(t)\right|_{H}^{2}+c_{2}\left|\Delta^{N}(t)\right|_{V}^{2}+2 \int_{0}^{t} c\left|\dot{\Delta}^{N}(\xi, 0)\right|^{2} d \xi \\
\leq & \delta_{1}^{N}(t)+\delta_{2}^{N}(t)+2 \epsilon\left|\Delta^{N}(t)\right|_{V}^{2} \\
& +\int_{0}^{t}\left\{\left|\dot{\Delta}^{N}(\xi, 0)\right|^{2}+\left(\frac{23}{2}+\left|b\left(q^{N}\right)\right|_{L^{\infty}}^{2}+\frac{1}{2}\left|\dot{a}\left(q^{N}\right)\right|_{L^{\infty}}^{2}\right)\left|\dot{\Delta}^{N}\right|_{H}^{2}\right. \\
& \left.+\left(2+\left|h\left(q^{N}\right)\right|_{L^{\infty}}^{2}+T^{2}\left|G\left(q^{N}\right)\right|_{L^{\infty}}^{2}\right)\left|\Delta^{N}\right|_{V}^{2}\right\} d \xi
\end{aligned}
$$

where

$$
\begin{aligned}
\delta_{1}^{N}(t)= & \int_{0}^{t}\left\{\left|a\left(q^{N}\right)\right|_{L^{\infty}}^{2}\left|\ddot{E}-\mathcal{P}^{N} \ddot{E}\right|_{H}^{2}\right. \\
& +\left(c_{1}^{2}+c^{2} K_{1}\right)\left|\dot{E}-\mathcal{P}^{N} \dot{E}\right|_{V}^{2}+|b(q)|_{L^{\infty}}^{2}\left|\dot{E}-\mathcal{P}^{N} \dot{E}\right|_{H}^{2} \\
& \left.+\left(|h(q)|_{L^{\infty}}^{2}+T^{2}|G(q)|_{L^{\infty}}^{2}\right)\left|E-\mathcal{P}^{N} E\right|_{H}^{2}\right\} d \xi \\
& +\frac{c_{1}^{2}}{\epsilon}\left|\mathcal{P}^{N} E(t)-E(t)\right|_{V}^{2}
\end{aligned}
$$




$$
\begin{aligned}
\delta_{2}^{N}(t)= & \left|a(q)-a\left(q^{N}\right)\right|_{L^{\infty}}^{2} \int_{0}^{t}|\ddot{E}|_{H}^{2} d \xi \\
& +\gamma^{2} \tilde{d}\left(q, q^{N}\right) \int_{0}^{t}|\dot{E}|_{V}^{2} d \xi+\left|b(q)-b\left(q^{N}\right)\right|_{L^{\infty}}^{2} \int_{0}^{t}\left|\mathcal{P}^{N} \dot{E}\right|_{H}^{2} d \xi \\
& +\left(\left|h(q)-h\left(q^{N}\right)\right|_{L^{\infty}}^{2}+T^{2}\left|G(q)-G\left(q^{N}\right)\right|_{L^{\infty}}^{2}\right) \times \\
& \int_{0}^{t}\left|\mathcal{P}^{N} E\right|_{H}^{2} d \xi+\frac{\gamma^{2}}{\epsilon} \tilde{d}\left(q, q^{N}\right)|E(t)|_{V}^{2} .
\end{aligned}
$$

Since $\epsilon>0$ is arbitrary, we may choose it to be such that $1>c_{2}-2 \epsilon>0$. Furthermore, the wave speed $c$ satisfies $2 c>>1$. We then use Assumptions A1)-A4) to claim that there exist constants $\nu_{1}, \nu_{2}>1$ and $1 \geq a_{0}>0$, independent of $q$, such that

$$
\begin{gathered}
a_{0}\left|\dot{\Delta}^{N}(t)\right|_{H}^{2}+\left(c_{2}-2 \epsilon\right)\left|\Delta^{N}(t)\right|_{V}^{2}+\int_{0}^{t}(2 c-1)\left|\dot{\Delta}^{N}(\xi, 0)\right|^{2} d \xi \\
\leq \delta_{1}^{N}(t)+\delta_{2}^{N}(t)+\int_{0}^{t} \nu_{1}\left|\dot{\Delta}^{N}\right|_{H}^{2}+\nu_{2}\left|\Delta^{N}\right|_{V}^{2} d \xi .
\end{gathered}
$$

Finally recalling the bounds on $\nu_{1}, \nu_{2}, a_{0}$, and $c_{2}-2 \epsilon$, we may rewrite the inequality as

$$
\begin{gathered}
a_{0}\left|\dot{\Delta}^{N}(t)\right|_{H}^{2}+\left(c_{2}-2 \epsilon\right)\left|\Delta^{N}(t)\right|_{V}^{2} \\
\leq \delta_{1}^{N}(t)+\delta_{2}^{N}(t)+\int_{0}^{t}\left(\frac{\nu_{2}}{c_{2}-2 \epsilon}\right) \nu_{1}\left|\dot{\Delta}^{N}\right|_{H}^{2}+\frac{\nu_{1} \nu_{2}}{a_{0}}\left|\Delta^{N}\right|_{V}^{2} d \xi \\
\leq \delta_{1}^{N}(t)+\delta_{2}^{N}(t)+\left(\frac{\nu_{1} \nu_{2}}{a_{0}\left(c_{2}-2 \epsilon\right)}\right) \int_{0}^{t} a_{0}\left|\dot{\Delta}^{N}\right|_{H}^{2}+\left(c_{2}-2 \epsilon\right)\left|\Delta^{N}\right|_{V}^{2} d \xi
\end{gathered}
$$

In order to apply Gronwall's inequality to obtain uniform convergence of $\Delta^{N}$ and $\dot{\Delta}^{N}$ in $t$ as $N \rightarrow \infty$, we must establish the uniform convergence of $\delta_{1}^{N}$ and $\delta_{2}^{N}$. We have from B3) and B4) that $\left|\ddot{E}(t)-\mathcal{P}^{N} \ddot{E}(t)\right|_{H},\left|\dot{E}(t)-\mathcal{P}^{N} \dot{E}(t)\right|_{V}$, and $\left|E(t)-\mathcal{P}^{N} E(t)\right|_{V}$ converge to zero as $N \rightarrow \infty$ for each $t$. Since this convergence is dominated and $\{E(t)\}_{t \in[0, T]}$ is compact in $V$, we have that

$$
\delta_{1}^{N} \rightarrow 0 \text { uniformly in } t \text { as } N \rightarrow \infty \text {. }
$$

Moreover, the boundedness of $E, \dot{E}$, and $\ddot{E}$ given by the enhanced regularity results and the assumption A8) imply that 


$$
\delta_{2}^{N} \rightarrow 0 \text { uniformly in } t \text { as } N \rightarrow \infty \text { and } q^{N} \rightarrow q \text { in } \tilde{Q} \text {. }
$$

Then we may apply Gronwall's inequality to conclude that

$$
\begin{aligned}
& \sup _{t \in[0, T]}\left|\Delta^{N}(t)\right|_{V}^{2} \rightarrow 0 \text { as } N \rightarrow \infty \\
& \sup _{t \in[0, T]}\left|\dot{\Delta}^{N}(t)\right|_{H}^{2} \rightarrow 0 \text { as } N \rightarrow \infty
\end{aligned}
$$

which is sufficient to prove the desired result.

\subsection{Estimation of parameters in the system with pressure-dependent Debye polarization}

The general system (10) is formulated to accomodate systems arising from a variety of electromagnetic interrogation problems. We are concerned here with a particular system that incorporates a pressure-dependent model for Debye polarization. We demonstrate that this system satisfies Assumptions A11), B1)-B3) and H1) and thus that the results of the previous section apply. (We note that verifications of Assumptions A1)-A10) are given in [9] and [24].)

The system we wish to consider is given by (10) with the parameter-dependent coefficients, kernel and forcing functions, and sesquilinear form (11).

For this system, the set of admissible parameters $Q$ is a subset of $\mathbb{R}^{7}$, where seven is the number of parameters to be estimated (in addition to the six polarization parameters from Section 2, one is often interested in estimating the conductivity coefficient $\sigma$ ). Here we consider $q \in Q \subset \mathbb{R}^{7}$ where $q=\left(\sigma, \gamma_{0}, \zeta_{0}, \tau_{0}, \kappa_{\gamma}, \kappa_{\zeta}, \kappa_{\tau}\right)$. We choose the admissible set $Q$ to insure that our Debye coefficients are well-defined. First, because of the physical meaning of these parameters, the values of $\sigma, \gamma_{0}, \zeta_{0}$ and $\tau_{0}$ must be positive. Then for a given pressure wave $p$ with $p, \dot{p} \in L^{\infty}\left(0, T ; L^{\infty}(0,1)\right)$ and a fixed $\delta>0$, we admit only values of $\kappa_{\gamma}, \kappa_{\zeta}$, and $\kappa_{\tau}$ such that $\gamma_{0}+\kappa_{\gamma} p(t, z), \zeta_{0}+\kappa_{\zeta} p(t, z)$, and $\tau_{0}+\kappa_{\tau} p(t, z)$ are greater than $\delta$ for all $z \in[0,1]$ and $t \in[0, T]$. In addition to these requirements, we assume that the admissible parameter set is closed and bounded in $\mathbb{R}^{7}$.

We recall that $q^{N} \rightarrow q$ in the standard Euclidean metric is equivalent to the convergence of each component of $q^{N}$. Moreover, any closed and bounded sets $Q$ in $\mathbb{R}^{7}$ are compact and satisfy the conditions of B1). The conditions B2)-B4) are satisfied by $V^{N}$, which is in this case the set of finite dimensional linear piecewise basis elements, and the projection operator $\mathcal{P}^{N}$. To verify H1), we note that $\sigma_{1}(q)(\phi, \psi)=c^{2}<\phi^{\prime}, \psi^{\prime}>$ is independent of $q$ and $\left|\sigma_{1}\left(q_{1}\right)(\phi, \psi)-\sigma_{1}\left(q_{2}\right)(\phi, \psi)\right|=0$ for any $q_{1}, q_{2} \in Q$. 
We next verify A11)i)-iv) for the coefficients in our model. We note that as $q^{N} \rightarrow q$ we have, for a given $p, \dot{p} \in L^{\infty}$,

$$
\begin{gathered}
\left|\sigma^{N}-\sigma\right| \rightarrow 0 \\
\left|\zeta_{0}^{N}+\kappa_{\zeta}^{N} p-\left(\zeta_{0}+\kappa_{\zeta} p\right)\right|_{L^{\infty}} \rightarrow 0 \\
\left|\gamma_{0}^{N}-\zeta_{0}^{N}+\left(\kappa_{\gamma}^{N}-\kappa_{\zeta}^{N}\right) p-\left(\gamma_{0}-\zeta_{0}+\left(\kappa_{\gamma}-\kappa_{\zeta}\right) p\right)\right|_{L^{\infty}} \rightarrow 0 \\
\left|\tau_{0}^{N}+\kappa_{\tau}^{N} p-\left(\tau_{0}-\kappa_{\tau} p\right)\right|_{L^{\infty}} \rightarrow 0 \\
\left|\left(\kappa_{\gamma}^{N}-\kappa_{\zeta}^{N}\right) \dot{p}-\left(\left(\kappa_{\gamma}-\kappa_{\zeta}\right) \dot{p}\right)\right|_{L^{\infty}} \rightarrow 0
\end{gathered}
$$

and

$$
\left|\kappa_{\tau}^{N} \dot{p}-\left(\kappa_{\tau} \dot{p}\right)\right|_{L^{\infty}} \rightarrow 0
$$

We use (20) directly to claim that $\left|a\left(q^{N}\right)-a(q)\right|_{L^{\infty}} \rightarrow 0$ whenever $q^{N} \rightarrow q$; thus A11)i) holds.

In demonstrating that A11)ii) holds, we observe that

$$
\begin{aligned}
& \left|b\left(q^{N}\right)-b(q)\right|_{L^{\infty}} \leq \frac{1}{\epsilon_{0}}\left|\sigma^{N}-\sigma\right| \\
& +\sup _{t \in[0, T]} \sup _{z \in\left[z_{1}, 1\right]}\left|\left(\frac{\gamma_{0}^{N}-\zeta_{0}^{N}+\left(\kappa_{\gamma}^{N}-\kappa_{\zeta}^{N}\right) p(t, z)}{\tau_{0}^{N}+\kappa_{\tau}^{N} p(t, z)}\right)-\left(\frac{\gamma_{0}-\zeta_{0}+\left(\kappa_{\gamma}-\kappa_{\zeta}\right) p(t, z)}{\tau_{0}+\kappa_{\tau} p(t, z)}\right)\right| .
\end{aligned}
$$

Then we may apply equations (19), (21), and (22) and the quotient rule of limits to conclude that $\left|b\left(q^{N}\right)-b(q)\right|_{L^{\infty}} \rightarrow 0$ as $q^{N} \rightarrow q$ and A11)ii) is satisfied.

From the definition of $h$, we have 


$$
\begin{gathered}
\left|h\left(q^{N}\right)-h(q)\right|_{L^{\infty}} \leq \sup _{t \in[0, T]} \sup _{z \in\left[z_{1}, 1\right]}\left\{\left|\frac{\left(\kappa_{\gamma}^{N}-\kappa_{\zeta}^{N}\right) \dot{p}(t, z)}{\tau_{0}^{N}+\kappa_{\tau}^{N} p(t, z)}-\frac{\left(\kappa_{\gamma}-\kappa_{\zeta}\right) \dot{p}(t, z)}{\tau_{0}+\kappa_{\tau} p(t, z)}\right|\right. \\
\left.+\left|\frac{\left(1+\kappa_{\tau} \dot{p}(t, z)\right)\left(\gamma_{0}-\zeta_{0}+\left(\kappa_{\gamma}-\kappa_{\zeta}\right) p(t, z)\right)}{\left(\tau_{0}+\kappa_{\tau} p(t, z)\right)^{2}}-\frac{\left(1+\kappa_{\tau}^{N} \dot{p}(t, z)\right)\left(\gamma_{0}^{N}-\zeta_{0}^{N}+\left(\kappa_{\gamma}^{N}-\kappa_{\zeta}^{N}\right) p(t, z)\right)}{\left(\tau_{0}^{N}+\kappa_{\tau}^{N} p(t, z)\right)^{2}}\right|\right\} .
\end{gathered}
$$

From equations (23), (22), (24), and (21) and the product and quotient rules of limits, we may conclude that A11)iii) holds.

To show that A11)iv) holds, we argue that

$$
\begin{aligned}
& \left|G\left(q^{N}\right)-G(q)\right|_{L^{\infty}} \leq \\
& \sup _{(t, s) \in[0, T] \times[0, T]} \sup _{z \in\left[z_{1}, 1\right]} \mid \exp \left(\int_{s}^{t} \frac{-d \xi}{\tau_{0}^{N}+\kappa_{\tau}^{N} p(\xi, z)}\right) \frac{\left(1+\kappa_{\tau}^{N} \dot{p}(s, z)\right)\left(\gamma_{0}^{N}-\zeta_{0}^{N}+\left(\kappa_{\gamma}^{N}-\kappa_{\zeta}^{N}\right) p(s, z)\right)}{\left(\tau_{0}^{N}+\kappa_{\tau}^{N} p(t, z)\right)^{2}\left(\tau_{0}^{N}+\kappa_{\tau}^{N} p(s, z)\right)^{2}} \\
& -\exp \left(\int_{s}^{t} \frac{-d \xi}{\tau_{0}+\kappa_{\tau} p(\xi, z)}\right) \frac{\left(1+\kappa_{\tau} \dot{p}(s, z)\right)\left(\gamma_{0}-\zeta_{0}+\left(\kappa_{\gamma}-\kappa_{\zeta}\right) p(s, z)\right)}{\left(\tau_{0}+\kappa_{\tau} p(t, z)\right)^{2}\left(\tau_{0}+\kappa_{\tau} p(s, z)\right)^{2}} \mid .
\end{aligned}
$$

We note that equation (22) coupled with the quotient rule for limits allows us to assert that

$$
\exp \left(\int_{s}^{t} \frac{-d \xi}{\tau_{0}^{N}+\kappa_{\tau}^{N} p(\xi, z)}\right) \rightarrow \exp \left(\int_{s}^{t} \frac{-d \xi}{\tau_{0}+\kappa_{\tau} p(\xi, z)}\right)
$$

as $q^{N} \rightarrow q$. Thus, we may use equations (24), (21), (22), and (25) with the quotient and product rules for limits to verify that

$$
\left|G\left(q^{N}\right)-G(q)\right|_{L^{\infty}} \rightarrow 0
$$

as $q^{N} \rightarrow q$.

We have thus verified that the theory established in Section 3.1 can be applied to the system corresponding to pressure-dependent Debye polarization described by the coefficients (11). 


\section{$4 \quad$ Numerical examples}

In the previous section, we demonstrated that a (sub)sequence of minimizers $\left\{\bar{q}^{N}\right\}$ of the cost functionals (15) converges to a minimizer $\bar{q}$ of (12). In this section we present computational results for the problem of finding $\bar{q}^{N}$ for a fixed $N$. (We attempt to choose $N$ large so that $\bar{q}^{N}$ is close to $\bar{q}$.)

This problem is equivalent to our main objective, estimating the polarization and conductivity parameters of a dielectric by comparing numerical solutions of the model with experimental data. We recall from Section 2 that our polarization model has six material-dependent parameters; in this section we fix $\sigma$ and consider only these six variables $\tau_{0}, \zeta_{0}, \gamma_{0}, \kappa_{\tau}, \kappa_{\zeta}$, and $\kappa_{\gamma}$ in the equation

$$
\left(\tau_{0}+\kappa_{\tau} p\right) \dot{P}+P=\epsilon_{0}\left(\gamma_{0}-\zeta_{0}+\left(\kappa_{\gamma}-\kappa_{\zeta}\right) p\right) E
$$

We want to test the feasibilty of estimating them from experimental data. At this time, we do not yet have data from experiments (an experimental device to obtain such data is currently being constructed); instead we create simulated data from our computations. The simulated data consists of the boundary data from a numerical approximation to the solution of the system with added noise. (See [9] for sample numerical solutions to the forward problem.) We compute this approximate solution with fixed parameters values. These values are then thought of as our "unknown" true material parameters. The goal is to estimate these values. We appraise our ability to solve the problem by comparing the estimates with the true fixed values. If we cannot accurately approximate the parameter values in this context, we cannot expect to be able to estimate them in an experimental setting.

We let $q$ generically denote the set of parameters we wish to estimate in the examples presented below; these may include the mean values in the polarization model, $\tau_{0}, \gamma_{0}$, and $\zeta_{0}$ and/or the coefficients of pressure in the polarization model, $\kappa_{\tau}, \kappa_{\gamma}$, and $\kappa_{\zeta}$. We let $q^{*}$ denote the true values of the corrsponding "unknown" parameters. We leave the values of all other parameters fixed.

There are two sets of electromagnetic reflections that reach the boundary. The first, after the initial signal, are the reflections from the air/dielectric interface and the second are from the virtual interface produced by the acoustic pressure wave. (Figure 4 depicts each set of reflections separately.) In some scenarios, using data that contains only one set of reflections may be advantageous. For example, one may use the data from the initial signal and the reflections from the air/dielectric interface (i.e., the first section of data in Figure 4) to refine the initial parameter estimates and then use these refinements with the data from the acoustic interface reflections to obtain final estimates. In another approach, one may use just the data from the acoustic interface reflections to estimate the parameters. In any case, the cost functional for the examples given here is of the form 


$$
J(q)=\sum_{i \in I}\left(E_{\text {data }}^{i}-E\left(t_{i}, 0 ; q\right)\right)^{2} .
$$

where $I$ corresponds to an appropriately chosen data set. (Since here we consider exclusively the finite dimensional system for a fixed $N$, we drop the $N$ for ease of notation.)
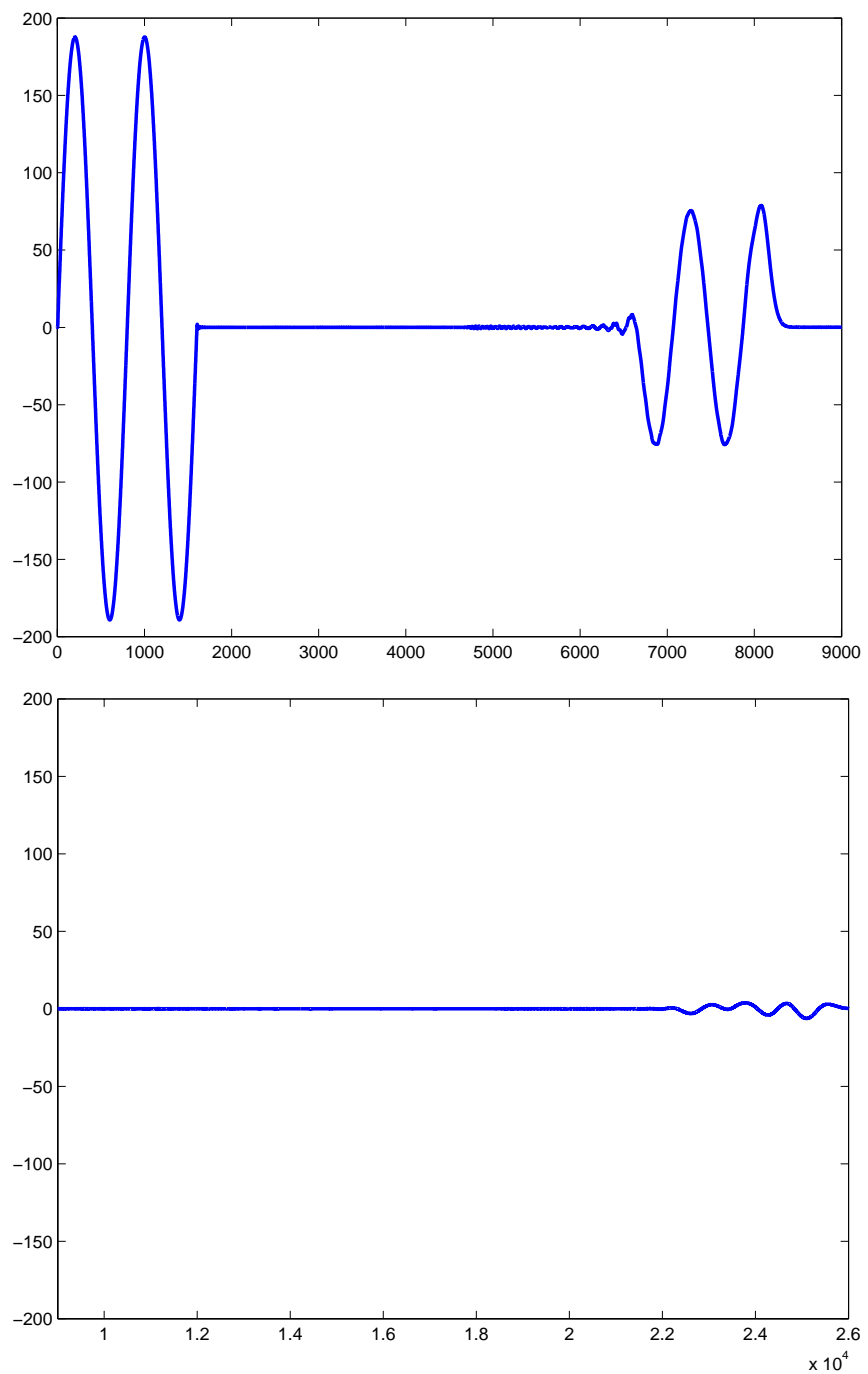

Figure 4: The two sets of reflections that reach the boundary

We used a Nelder Mead optimization routine [19] to find the parameter values that minimize the cost function. This optimization method is a gradient-free, simplex search method. The use of a gradient-based method to solve this problem is impractical due to computational 
time. The particular routine used requires an initial simplex of estimates and a termination tolerance for the difference between subsequent function evaluations. We choose the initial estimates to have varying levels of error in relation to the true parameter values. For these computations, we set the termination tolerance at 1e-09.

As already noted, we created simulated data to test our algorithms. The data set without noise is simply observations at the boundary taken from a forward simulation of the model using the parameter set $q^{*}$. The data sets with error were created by adding an appropriate amount of normally distributed relative random noise to the original data set. The random noise was generated by the MATLAB command randn which creates normally distributed noise with mean 0 and variance 1 and was scaled and shifted appropriately. Because the noise is relative, the magnitude of noise is greater in the intervals of data that contain the initial interrogating impulse and the reflections.

We next present sample results for specific parameter estimation problems. We first consider the problem of estimating $q^{*}=\left[\gamma_{0}^{*}, \zeta_{0}^{*}, \lambda^{*}=\frac{1}{\sqrt{\mu_{0} \epsilon_{0} \tau_{0}^{*}}}\right]=[78.2,5.5,0.10545728042059]$ from data with varying levels of noise. Here $\tau_{0}^{*}$ is so small that it is advantageous to estimate $\lambda^{*}$, a scaled function of $\tau_{0}^{*}$; an estimated value of $\tau_{0}^{*}$ may be computed from an estimation of $\lambda^{*}$. We use the data containing the initial signal and the reflections from the air/dielectric interface to refine the initial parameter estimates $q_{0}$ and the data containing the reflections from the acoustic interface to obtain final estimates. We present the results in the following table. 


\begin{tabular}{|l|l|}
\hline Initial estimate $q_{0}$ & Final estimate for data without noise $\bar{q}$ \\
\hline \hline$q_{0}=1.0 q^{*}$ & {$[78.2,5.5,0.10545728042059]$} \\
\hline$q_{0}=0.95 q^{*}$ & {$[78.190631,5.499999,0.105462]$} \\
\hline$q_{0}=1.05 q^{*}$ & {$[78.197841,5.500000,0.105458]$} \\
\hline$q_{0}=0.9 q^{*}$ & {$[77.128609,5.499937,0.107040]$} \\
\hline$q_{0}=1.1 q^{*}$ & {$[70.763992,5.499504,0.117540]$} \\
\hline \hline & Final estimate for data with $1 \%$ noise \\
\hline \hline$q_{0}=0.95 q^{*}$ & {$[78.210472,5.499997,0.105444]$} \\
\hline$q_{0}=1.05 q^{*}$ & {$[77.260485,5.499940,0.106852]$} \\
\hline$q_{0}=0.9 q^{*}$ & {$[78.198944,5.499998,0.105461]$} \\
\hline$q_{0}=1.1 q^{*}$ & {$[78.482467,5.500010,0.105052]$} \\
\hline \hline & Final estimate for data with 5\% noise \\
\hline \hline$q_{0}=0.95 q^{*}$ & {$[74.876211,5.499764,0.110559]$} \\
\hline$q_{0}=1.05 q^{*}$ & {$[77.975797,5.499939,0.105722]$} \\
\hline$q_{0}=0.9 q^{*}$ & {$[78.337467,5.500010,0.105186]$} \\
\hline$q_{0}=1.1 q^{*}$ & {$[78.405972,5.499987,0.105175]$} \\
\hline \hline
\end{tabular}

Table 1: Parameter estimation results for $q^{*}=\left[\gamma_{0}^{*}, \zeta_{0}^{*}, \lambda^{*}=\frac{1}{\sqrt{\mu_{0} \epsilon_{0}} \tau_{0}^{*}}\right]=[78.2,5.5,0.10545728042059]$

These results illustrate that it is possible to recover accurate approximations of $\gamma_{0}^{*}, \zeta_{0}^{*}$, and $\lambda^{*}$ in the presence of noise and with error up to $10 \%$ in the initial estimates. A few of the results are unexpected, for instance the ability to approximate the values better in the presence of $5 \%$ noise with an initial guess with $-10 \%$ error than with an initial guess with $-5 \%$ error. We suspect these anomalies are due to the simplex search nature of the optimization routine.

The results in the tables clearly indicate that we can recover $q^{*}$ without much error. However it is often illustrative to compare the solutions calculated with the estimates with the solutions calculated with $q^{*}$. To do this, we plot the absolute value of the error for the solutions computed at the boundary, i.e.,

$$
\left|E\left(t_{i}, 0 ; \bar{q}\right)-E\left(t_{i}, 0 ; q^{*}\right)\right|
$$

where $\bar{q}$ is the final estimate (given in the table). As an example, Figure 5 depicts this error for the estimation problem with $5 \%$ noise and an initial guess with $-10 \%$ error. We see that overall the magnitude of error is small and that, as expected, the most error occurs in approximating the material and acoustic interface reflections. 


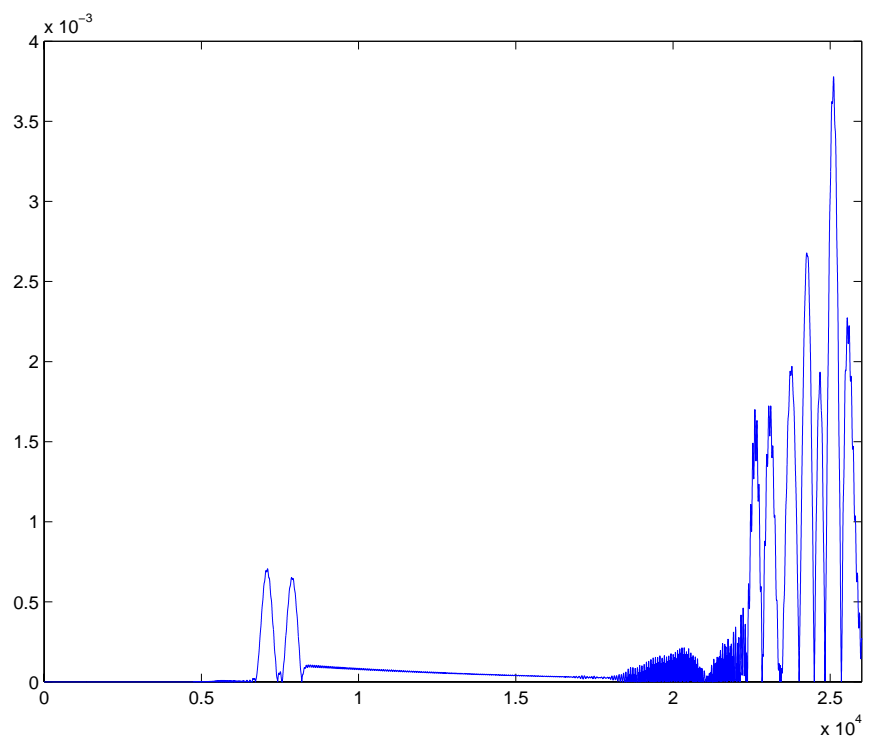

Figure 5: $\left|E\left(t_{i}, 0 ; \bar{q}\right)-E\left(t_{i}, 0 ; q^{*}\right)\right|$ vs $t_{i}-$ Absolute error for the parameter estimation problem with $5 \%$ noise and an initial guess with $-10 \%$ error.

We next consider the estimation of $q^{*}=\left[\kappa_{\gamma}^{*}, \kappa_{\zeta}^{*}, \kappa_{\tau}^{*}\right]=[46.92,1.65,1.581139 e-09]$ from the previous data sets. Since these parameters are the coefficients of pressure, they are irrelevant and undeterminable until the electromagnetic/acoustic interaction occurs. Thus we include only the second section of data in the cost functional. We present the results below. 


\begin{tabular}{|l|l|}
\hline Initial estimate $q_{0}$ & Final estimate for data without noise $\bar{q}$ \\
\hline \hline$q_{0}=0.99 q^{*}$ & {$[46.450800,1.633500,3.16174 \mathrm{e}-09]$} \\
\hline$q_{0}=1.01 q^{*}$ & {$[46.92,1.65,1.58114 \mathrm{e}-09]$} \\
\hline$q_{0}=0.95 q^{*}$ & {$[44.574000,1.567500,5.52700 \mathrm{e}-09]$} \\
\hline$q_{0}=1.05 q^{*}$ & {$[49.266000,1.732500,0]$} \\
\hline$q_{0}=0.9 q^{*}$ & {$[42.228000,1.485000,0]$} \\
\hline$q_{0}=1.1 q^{*}$ & {$[51.61200,1.815000,0]$} \\
\hline \hline & Final estimate for data with 1\% noise \\
\hline \hline$q_{0}=0.99 q^{*}$ & {$[46.450800,1.633500,3.17078 \mathrm{e}-09]$} \\
\hline$q_{0}=1.01 q^{*}$ & {$[47.389200,1.666500,-7.2435 \mathrm{e}-10]$} \\
\hline$q_{0}=0.95 q^{*}$ & {$[44.574000,1.567500,5.53493 \mathrm{e}-09]$} \\
\hline$q_{0}=1.05 q^{*}$ & {$[49.266000,1.732500,-1.064074 \mathrm{e}-08]$} \\
\hline$q_{0}=0.9 q^{*}$ & {$[42.228000,1.485000,6.01095 \mathrm{e}-09]$} \\
\hline$q_{0}=1.1 q^{*}$ & {$[51.612000,1.814500,-1.712928]$} \\
\hline \hline & Final estimate for data with 5\% noise \\
\hline \hline$q_{0}=0.99 q^{*}$ & {$[46.450800,1.633500,3.22126 \mathrm{e}-09]$} \\
\hline$q_{0}=1.01 q^{*}$ & {$[47.389200,1.666500,-6.6658 \mathrm{e}-10]$} \\
\hline$q_{0}=0.95 q^{*}$ & {$[44.574000,1.567500,5.56261 \mathrm{e}-09]$} \\
\hline$q_{0}=1.05 q^{*}$ & {$[49.266000,1.732500,-1.061871 \mathrm{e}-08]$} \\
\hline$q_{0}=0.9 q^{*}$ & {$[42.228000,1.485000,6.02759 \mathrm{e}-09]$} \\
\hline$q_{0}=1.1 q^{*}$ & {$[51.612000,1.815000,-1.712024 \mathrm{e}-08]$} \\
\hline \hline
\end{tabular}

Table 2: Parameter estimation results for $q^{*}=\left[\kappa_{\gamma}^{*}, \kappa_{\zeta}^{*}, \kappa_{\tau}^{*}\right]=[46.92,1.65,1.581139 e-09]$

We are able to obtain reasonable estimates for the parameters, especially $\kappa_{\gamma}^{*}$ and $\kappa_{\zeta}^{*}$. We have difficulty estimating the value of $\kappa_{\tau}^{*}$, most likely because the value is small. In general, the estimation error increases with the error in the initial guess. However, increasing the noise level in the data does not significantly effect the estimation accuracy.

We note that we obtain better estimates for the mean values $\left(\gamma_{0}^{*}, \zeta_{0}^{*}\right.$, and $\left.\tau_{0}^{*}\right)$ than for the pressure coefficients $\left(\kappa_{\gamma}^{*}, \kappa_{\zeta}^{*}\right.$, and $\left.\kappa_{\tau}^{*}\right)$. This is understandable, as the mean values are more influential in the system dynamics. They are also more important in identifying and characterizing the material.

In an electromagnetic interrogation parameter estimation problem, an estimate is sufficient if it can be used to classify the material. We consider the results for estimating $q^{*}=\left[\kappa_{\gamma}^{*}, \kappa_{\zeta}^{*}, \kappa_{\tau}^{*}\right]=[46.92,1.65,1.581139 e-09]$ using data with $5 \%$ relative normal noise and an initial guess with $-10 \%$ error. After solving the parameter estimation problem, we obtain the result $\bar{q}=[42.228000,1.485000,6.02759 e-09]$. If parameter values within the hypothet- 
ical range, $40<\kappa_{\gamma}<50,1.4<\kappa_{\zeta}<1.8$, and $1 e-09<\kappa_{\tau}<9 e-09$ are characteristic of the material under interrogation, we are successful in our attempt to solve the estimation problem. On the other hand, if the characteristic material parameters fall within the (hypothetical) range $45<\kappa_{\gamma}<47,1.6<\kappa_{\zeta}<1.7$, and $1 e-09<\kappa_{\tau}<2 e-09$, we are unable to characterize the material with our estimates and our attempt is unsuccessful. Ranges of these parameter values for different materials have not been experimentally determined, so we have no concrete measure as yet to assess our ability to solve the problem.

\section{Concluding remarks}

We have presented theoretical and computational results for a new class of inverse problems arising in nondestructive interrogation of materials. Our focus is on reflections from acoustic pressure waves that are moving through dielectric material targets. A detailed atomic based model for acoustic-dependence of dielectric parameters in a Debye material was given and this was incorporated into a theoretical framework for both forward solutions and least squares parameter estimation.

Computational findings suggest that primary parameters (relaxation, static permittivity, etc.) will be readily identifiable while aspects of the nonlinear dependence on pressure (first order coefficients) may be ascertained if appropriate data is available.

Our efforts on this methodology are continuing. An experimental device (similar to that depicted in Figure 7.1 of [4]) is currently under construction. Data from this device will be used to test and validate the methods developed in this paper.

\section{Acknowledgements}

This research was supported in part by the Air Force Office of Scientific Research under grants AFOSR-F49620-01-1-0026, and AFOSR-F49620-98-1-0430 and in part through a Department of Education GAANN Fellowship to J. K. Raye under Grant P200A70707.

\section{References}

[1] R. A. Albanese, J. Penn, and R. Medina, "Short-rise-time microwave pulse propagation through dispersive biological media", J. Optical Society of America A 6 (1989), pp. 1441-1446.

[2] J. C. Anderson, Dielectrics, Reinhold Publishing Company, New York, 1964. 
[3] C. A. Balanis, Advanced Engineering Electromagnetics, John Wiley \& Sons, New York, 1989.

[4] H. T. Banks, M. W. Buksas, and T. Lin, Electromagnetic Material Interrogation Using Conductive Interfaces and Acoustic Wavefronts, SIAM Frontiers in Applied Mathematics, Philadelphia, 2000.

[5] H.T. Banks, M.L. Joyner, B. Wincheski, and W.P. Winfree, "Electromagnetic interrogation techniques for damage detection", CRSC-TR01-15, NCSU, June 2001; Proceedings Electromagnetic Nondestructive Evaluation 2001, Kobe, Japan, May 18-19, 2001, IOS Press, Amsterdam, (2002), to appear.

[6] H.T. Banks, M.L. Joyner, B. Wincheski, and W.P. Winfree, "Real time computational algorithms for eddy current based damage detection", CRSC-TR01-16, NCSU, June 2001; Inverse Problems, to appear.

[7] H.T. Banks, M.L. Joyner, B. Wincheski, and W.P. Winfree, "Nondestructive evaluation techniques using a reduced order computational methodology", ICASE Technical Report 2000-10, NASA Langley Research Center, March 2000; Inverse Problems, 16 (2000), pp. 929-945.

[8] H. T. Banks, C. J. Musante, and J. K Raye, "Approximate methods for inverse problems governed by nonlinear parabolic systems", Numerical Functional Analysis and Optimization, 21 (2000), pp 791-816.

[9] H. T. Banks and J. K. Raye, "Well-posedness for systems representing electromagnetic/ acoustic wavefront interaction", CRSC-TR01-34, December 2001; ESIAM: Control, Optimization, and Calculus of Variations, to appear.

[10] H. T. Banks and J. K. Raye, "Computational methods for nonsmooth acoustic systems", CRSC-TR01-02, NCSU, January 2001; Computational and Applied Mathematics, to appear.

[11] H. T. Banks, R. C. Smith, and Y. Wang, Smart Material Structures: Modeling, Estimation, and Control, J. Wiley \& Sons, Chichester, 1996.

[12] H. T. Banks and J. Zhou, "Regularity and approximation of systems arising in electromagnetic interrogation of dielectric materials", Num. Func. Analysis and Optimization, 20 (1999), pp. 609-627.

[13] G. K. Batchelor, An Introduction to Fluid Dynamics, Cambridge University Press, Cambridge, 1967.

[14] S. R. deGroot and P. Mazur, Non-equilibrium Thermodynamics, North-Holland Publishing Company, Amsterdam, 1962. 
[15] R. S. Elliott, Electromagnetics: History, Theory, and Applications, IEEE Press, New York, 1993.

[16] F. Franks, Water A Comprehensive Treatise, Plenum Press, New York, 1972.

[17] E. H. Grant, R. J. Sheppard, and G. P. South, Dielectric Behavior of Biological Models in Solution, Clarendon Press, Oxford, 1978.

[18] J. D. Jackson, Classical Electrodynamics, John Wiley \& Sons, New York, 2nd edition, 1975 .

[19] C. T. Kelley, Iterative Methods for Optimization, SIAM Frontiers in Applied Mathematics, Philadelphia, 1999.

[20] D. K. Kondepudi and I. Prigogine, Modern Thermodynamics From Heat Engines to Dissipative Structures, John Wiley \& Sons, New York, 1998.

[21] N. H. March and M. P. Tosi, Atomic Dynamics in Liquids, Dover, New York, 1976.

[22] P. M. Morse and K. U. Ingard, Theoretical Acoustics, The McGraw-Hill Companies, Inc., New York, 1968.

[23] R. H. Nochetto and C. Verdi, "Approximations of degenerate parabolic problems using numerical integration, SIAM Journal on Numerical Analysis, 25 (1988), pp. 784-814.

[24] J. K. Raye, An Electromagnetic Interrogation Technique Utilizing Pressure-Dependent Polarization, Ph.D. Thesis, North Carolina State University, in preparation.

[25] J. A. Stratton, Electromagnetic Theory, The McGraw-Hill Companies, Inc., New York, 1941.

[26] J. H. Van Vleck, The Theory of Electric and Magnetic Susceptibilities, Oxford University Press, London, 1931. 